Historic, Archive Document

Do not assume content reflects current scientific knowledge, policies, or practices. 



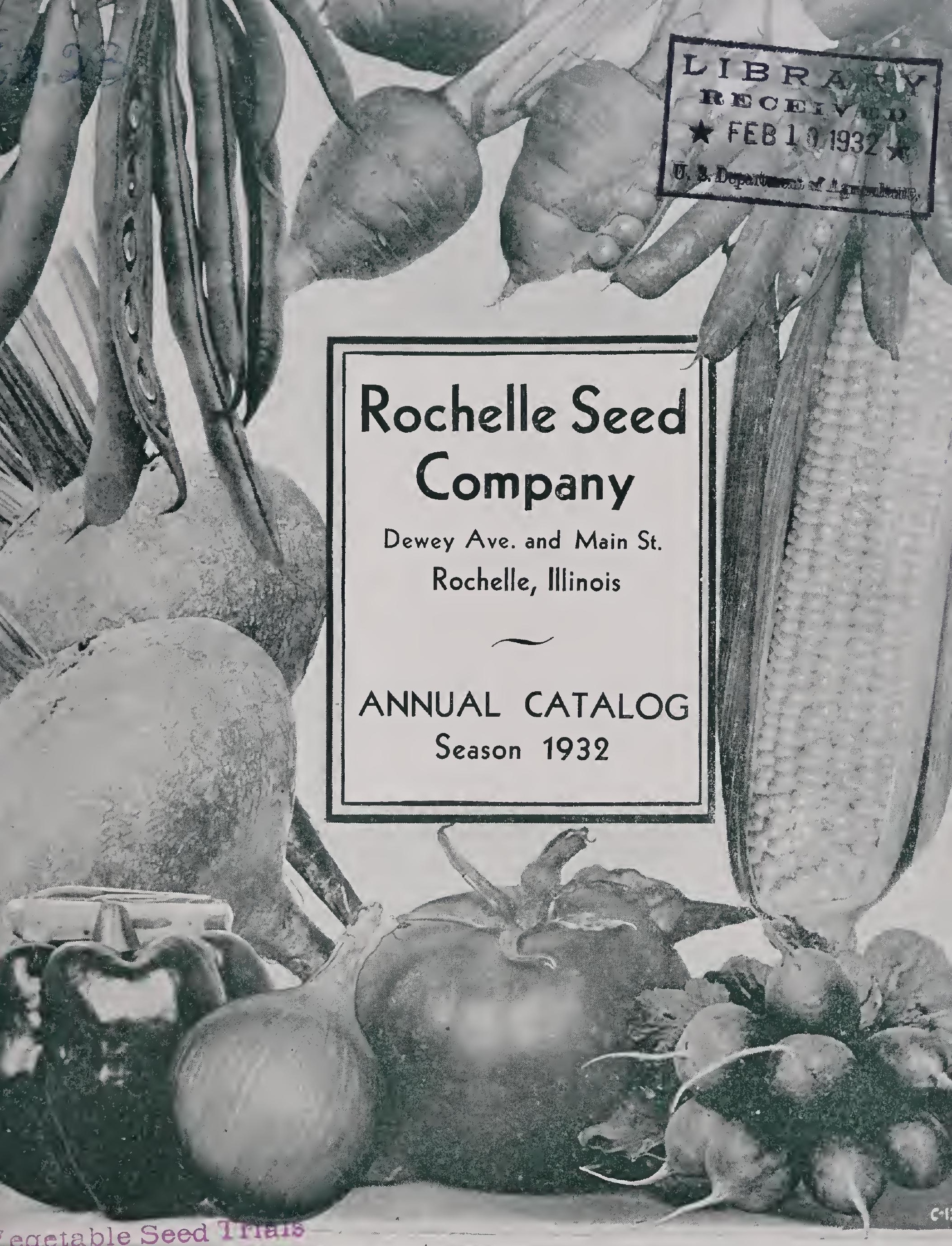
egretable Seed Trats

\section{Rochelle Seed
Company}

Dewey Ave. and Main St.

Rochelle, Illinois

Season 1932 



\section{Information for Purchasers}

OUR ITEMS ARE STRICTLY CASH WITH ORDER. We do nothing C. O. D.

Every item entering into the seed business is strictly spot cash. Cash buys the best of everything. When cost of seed, postage and other necessary expense is paid, high grade seeds are sold on a very small margin of profit. It is not big profits in a single order, but the large number of orders we receive enables us to make the low prices and give you the most possible for your money.

HOW TO SEND MONEY. Remittance may be sent at our risk by any of the following methods, viz: Postal Money Order, Draft on New York or Chicago, or Express Company Money Order.

WE PAY POSTAGE on all vegetable or flower seed quoted by the packet, ounce or pound (except where otherwise noted) and guarantee safe delivery at any post office of the United States, provided that orders for peas, beans and corn shall also include a reasonable amount of other flower and vegetable seeds.

IT MATTERS NOT HOW SMALL YOUR ORDER, nor what you may want in the seed line, we shall be glad to hear from you even for one packet; it will have our best attention. Our ambition is to serve you in a manner that will make you one of our satisfied customers.

GUARANTEE. Complaints made that seeds are not good should quite as often be attributed to other causes as to the quality of the seeds. There are hundreds of contingents constantly arising to prevent the best of seeds giving satisfaction, such as sowing too deep or too shallow, in too wet or dry soil, insects of all kinds destroying the plants as soon as they appear, wet weather, cold weather, frosts, chemical changes in the seeds induced by temperature, etc. For the above reasons it is impractical to guarantee seeds under all circumstances, yet at the same time, we exercise the greatest care in seeing that our seeds are fresh and true to name, knowing that your success is ours, and that a satisfied customer is our best advertisement. Therefore we do not in any way, express or implied, guarantee the description, quality, productiveness or any other matter of any seeds sold by us, and we will not in any way be responsible for the crop. If the purchaser does not accept these goods on the above terms, no sale is made thereof, and he must return them at once and money will be refunded.

PRICES. In comparing our mail prices, especially on heavy seeds such as peas, beans and corn, by the packet, ounce or pound, BEAR IN MIND WE PAY.THE POSTAGE. All we ask is that you give us your order for what light seeds you may need.

SPECIAL PRICES TO MARKET GARDENERS. Those who make a business of raising vegetables for the market, and who require large quantities of seeds are entitled to lower prices, owing to the fact that the expense of measuring and putting up the seeds in small packages is done away with. If you desire large amounts of seeds, we should advise you to write for special quotations on the quantities desired before placing your order.

ORDER EARLY. It is important to order as soon after you get this catalogue as convenient, then you will have your seeds on hand for planting when you want them. Besides if you order early, you have a chance to test your seeds and satisfy yourself they are of good germination before planting them.

ERRORS. The utmost care is taken in our house to fill orders accurately, but if an error occurs, as will sometimes be the case in any business house, please notify us at once, and it will be promptly and cheerfully corrected. Shortage or other loss, however, must be reported at once upon receipt of goods or no claims will be allowed.

OUR SEED TRADE is entirely through this catalogue. We send no commission seeds, but devote all our endeavors to supplying our trade with the finest stock obtainable. The substantial increase in our business each year assure us that our efforts are not in vain.

\section{Rochelle Seed Company}

\section{Rochelle, Illinois}

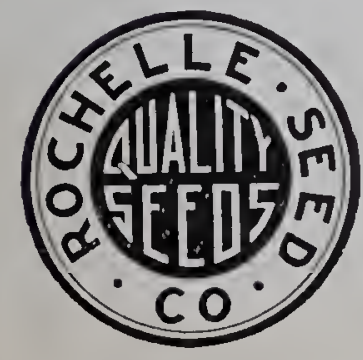

Our office and warehouse is located at Main Street and Dewey Avenue (Just north of C. \& N. W. Depot). Come to see us. Phone Number 252. Our facilities to serve you are greatly improved. You'll like our service.

Free Seeds On orders amounting to $\$ 2.00$ or over, you may to garden and flower seeds. 


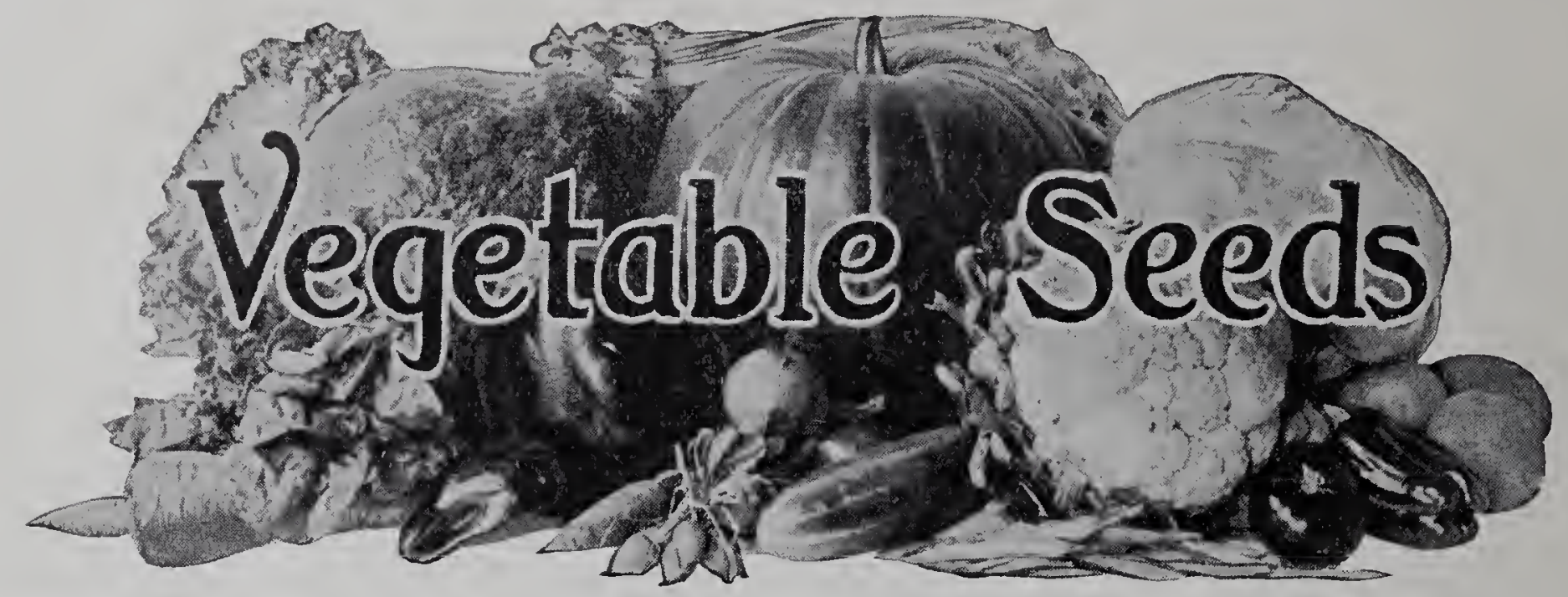

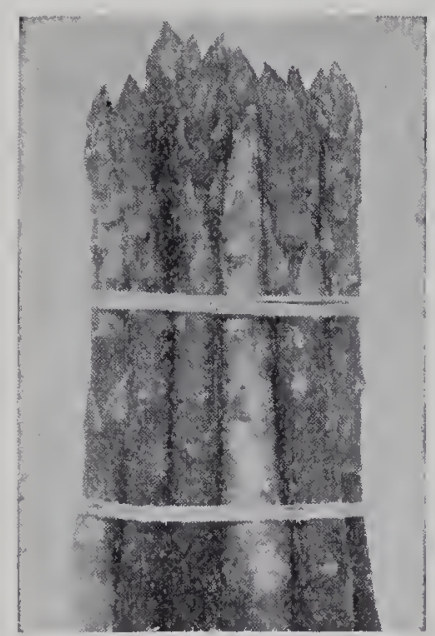

Palmetto Asparagus

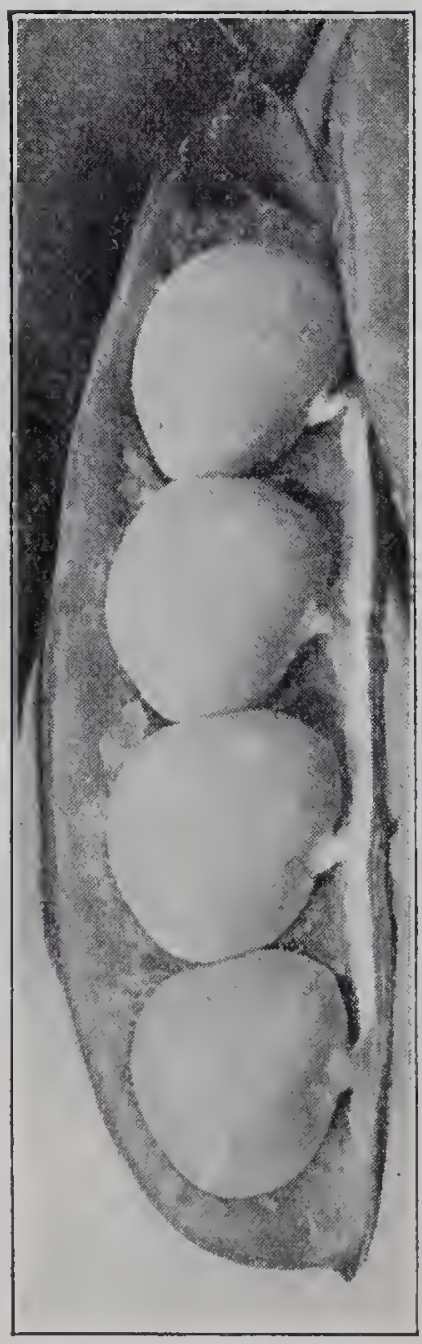

Henderson's Bush Lima

\begin{abstract}
ASPARAGUS
Washington

Conover's Colossal

Palmetto

BEANS-Wax or Early Pod'd Bush

Davis Kidney Wax.

Golden Wax

Wardwell's Kidney Wax

Black Wax

Webber Wax

Currie's Rust Proof.

(Green Podded Varieties)

$$
\begin{array}{lll}
1 / 2 & 1 & 2
\end{array}
$$

Stringless Pkt. Lb. Lb. Lbs. $\begin{array}{lllll}\text { Green Pod.... } & .10 & .25 & .45 & .80\end{array}$

$\begin{array}{llllll}\text { Longfellow } & \ldots . . & .10 & .25 & .45 & .80\end{array}$

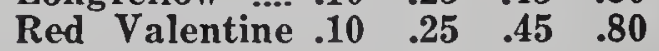

\section{POLE BEANS}

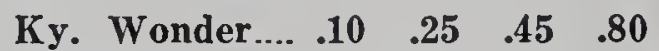

Speckled

$\begin{array}{lllll}\text { Cranberry .... } & .10 & .25 & .45 & .80\end{array}$

Lazy Wife ...... $.10 \quad .25 \quad .45 \quad .80$
\end{abstract}

Pkt.

.10

.05

\section{DWARF LIMA BEANS}

Henderson's Bush .10

Imp. Bush Lima.. .10

.25

.45

\section{POLE LIMA BEANS}

Seibert's Early

$\begin{array}{rrrrr}\text { Lima........... } & .10 & .25 & .45 & .80 \\ \text { Ideal Pole Lima... } & .10 & .25 & .45 & .80 \\ \text { King of Garden.... } & .10 & .25 & .45 & .80\end{array}$

\section{FIELD BEANS}

Navy

Red Kidney

BEETS

Pkt. Oz. $1 / 21$

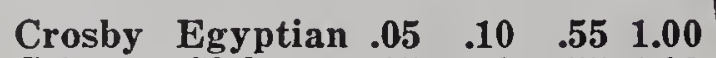

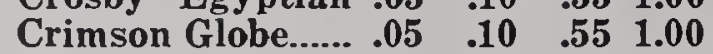

Ex. Early Egypt'n $.05 \quad .10 \quad .55 \quad 1.00$

Half Long Red....

$\begin{array}{llll}\text { Detroit Dark Red } .05 & .10 & .55 & 1.00\end{array}$

$\begin{array}{rrrr}\text { Oz. } & 1 / 2 \text { Lb. } & \text { 1 Lb. } & 2 \text { Lbs. } \\ .20 & \ldots \ldots . & \ldots \ldots . & \ldots \ldots . \\ .10 & \ldots \ldots . . & \ldots \ldots . . & \ldots \ldots . \\ .10 & \ldots \ldots . & \ldots \ldots . & \ldots \ldots \\ & & & \\ \ldots \ldots . & .25 & .45 & .80 \\ \ldots . . & .25 & .45 & .80 \\ \ldots \ldots & .25 & .45 & .80 \\ \ldots \ldots . . & .25 & .45 & .80 \\ \ldots . . & .25 & .45 & .80 \\ \ldots \ldots & .25 & .45 & .80\end{array}$

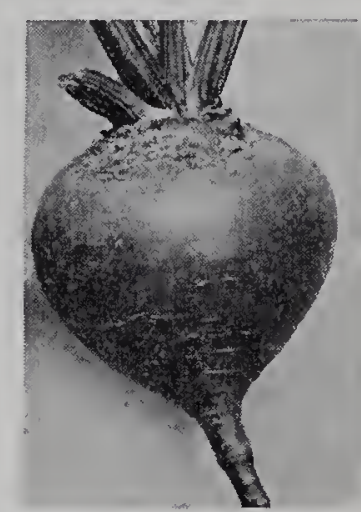

Detroit Dark Red Beet

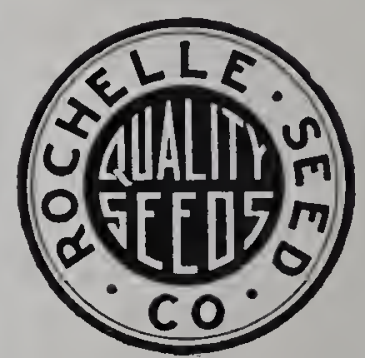

PLANT ROCHELLE QUALITY SEEDS

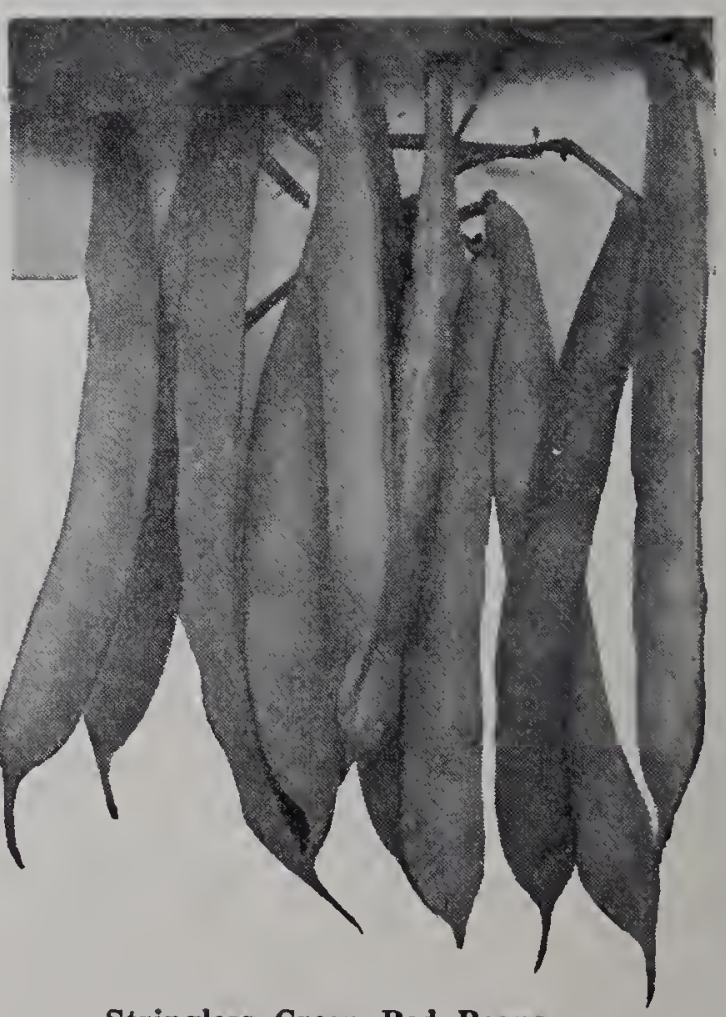

Stringless Green Pod Beans 


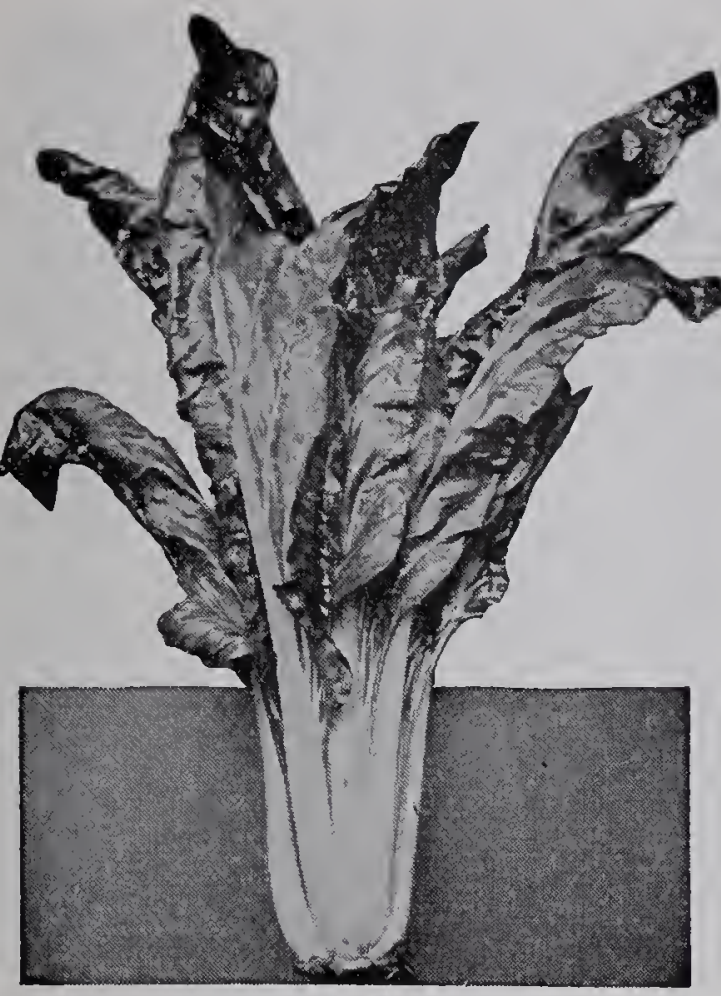

Lucullus Swiss Chard

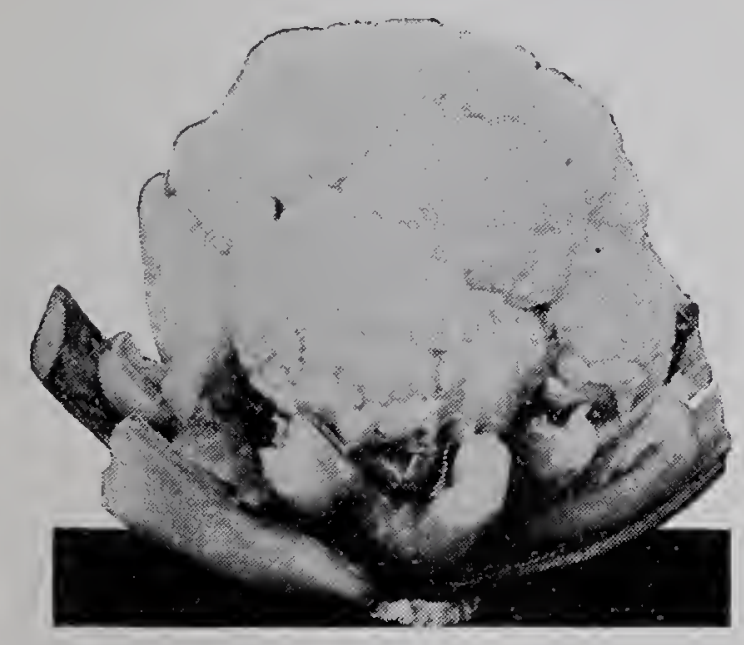

Early Snowball Cauliflower

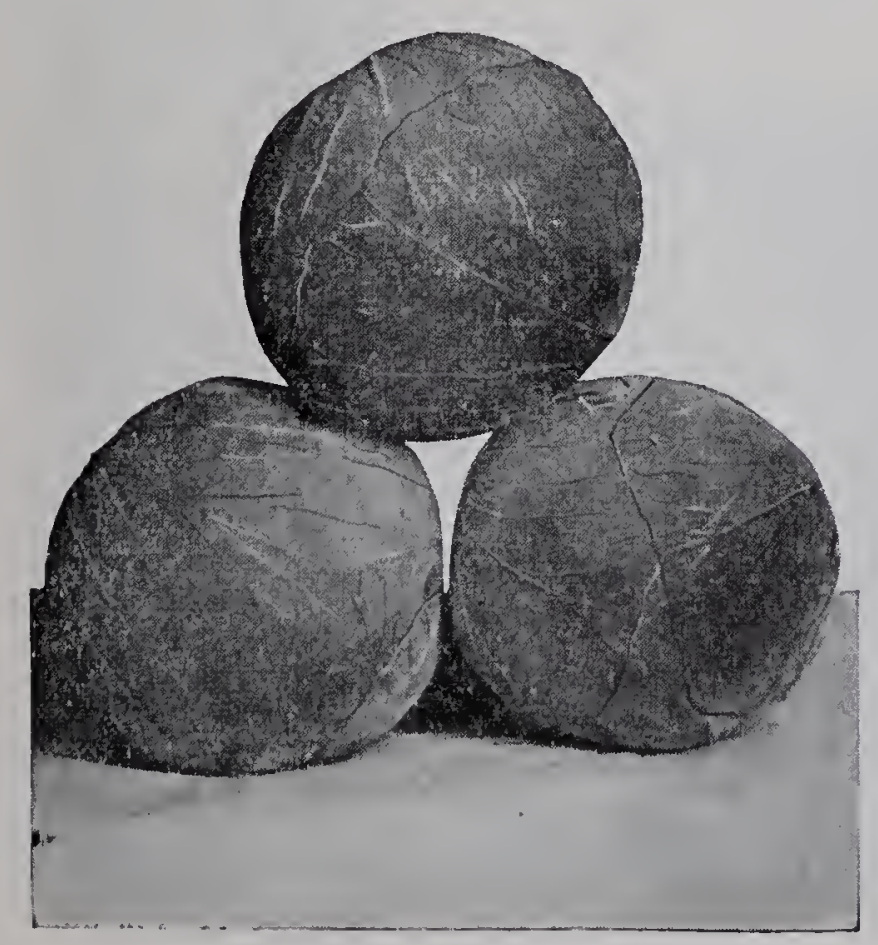

Hollander or Ball Head

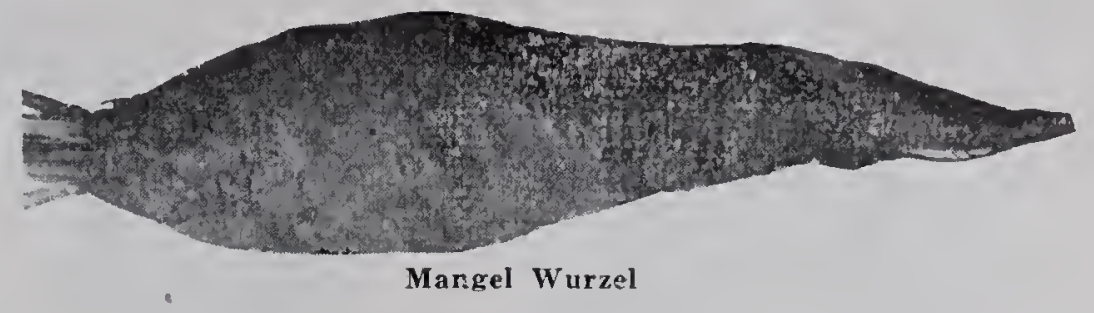

SWISS CHARD

Pkt. Oz. $1 / 2$ Lb. $1 \mathrm{Lb}$.

Silver Leaf $.05 \quad .10$

Lucullus

$.05 \quad .10$

CATTLE BEETS or MANGEL WURZELS

$\begin{array}{lllll}\text { Giant Half Sugar.......................... } & \ldots \ldots . . & .40 & .75 \\ \text { Long Red ..................................... } & . . . . & .40 & .75\end{array}$

BRUSSELS SPROUTS ........... .05 $\quad .10$

\section{CABBAGE}

Early Winningstadt................05

Wakefield ................................ .05

Early Summer .......................... .05

Copenhagen Market................ .05 .25

Sure Header ............................ $.05 \quad .25$

Fottler's Brunswick................ .05

Rock Red .................................. .05 .25

Premium Flat Dutch.............. .05 .25

All Seasons .............................. .05

Hollander or Ball Head........ .05 .25

Celery Cabbage....................... .05 $\quad .25$

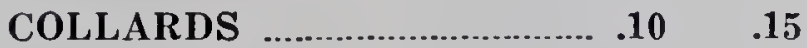

\section{CARROTS}

Oxheart

Early Scarlet Horn

Chantenay

Long Orange

Danvers

$\begin{array}{lll}.10 & \ldots . . & \ldots . . \\ .10 & \ldots . . & \ldots . . \\ .10 & \ldots . . & \ldots . . \\ .10 & \ldots . . & \ldots . . \\ .10 & \ldots . . . & \ldots . .\end{array}$

\section{STOCK CARROTS}

Mammoth White Belgian...... $.05 \quad .10 \quad .45 \quad .85$ Mammoth Yellow Belgian.... $.05 \quad \mathbf{0 5} \quad .45$

\section{CAULIFLOWER}

Early Paris ........................... 10

Early Snowball ....................... .10

Dwarf Erfurt

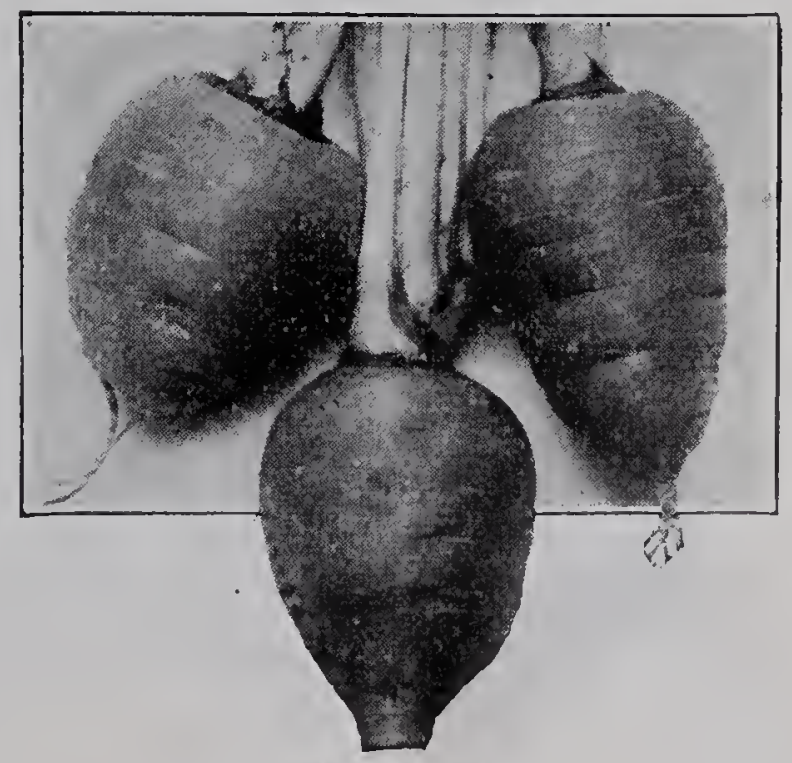

Oxheart Carrots 


\section{CELERY}

White Plume

Golden Self-Blanching.

Golden Heart

Giant Pascal
CELERIAC

Giant Prague CRESS

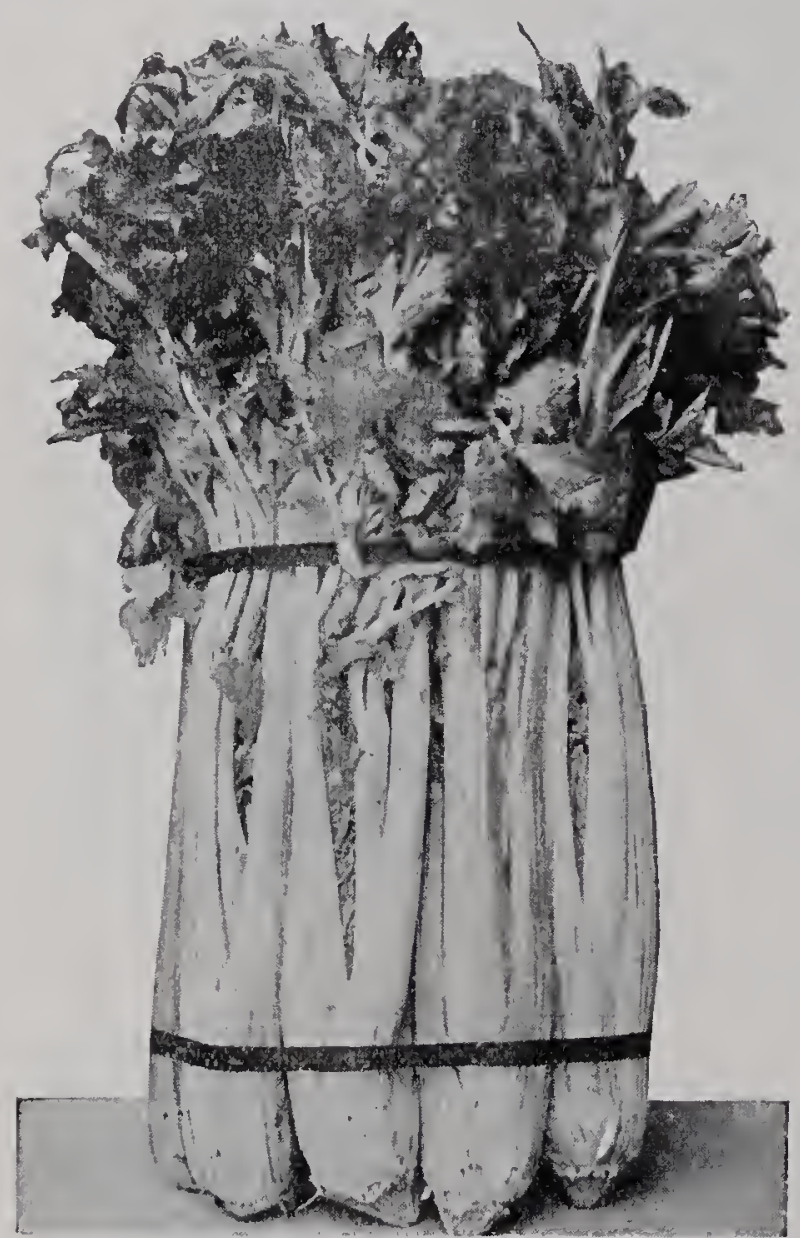

Golden Self-Blanching Celery
Pkt.

.05

.10

.05

.05

.05

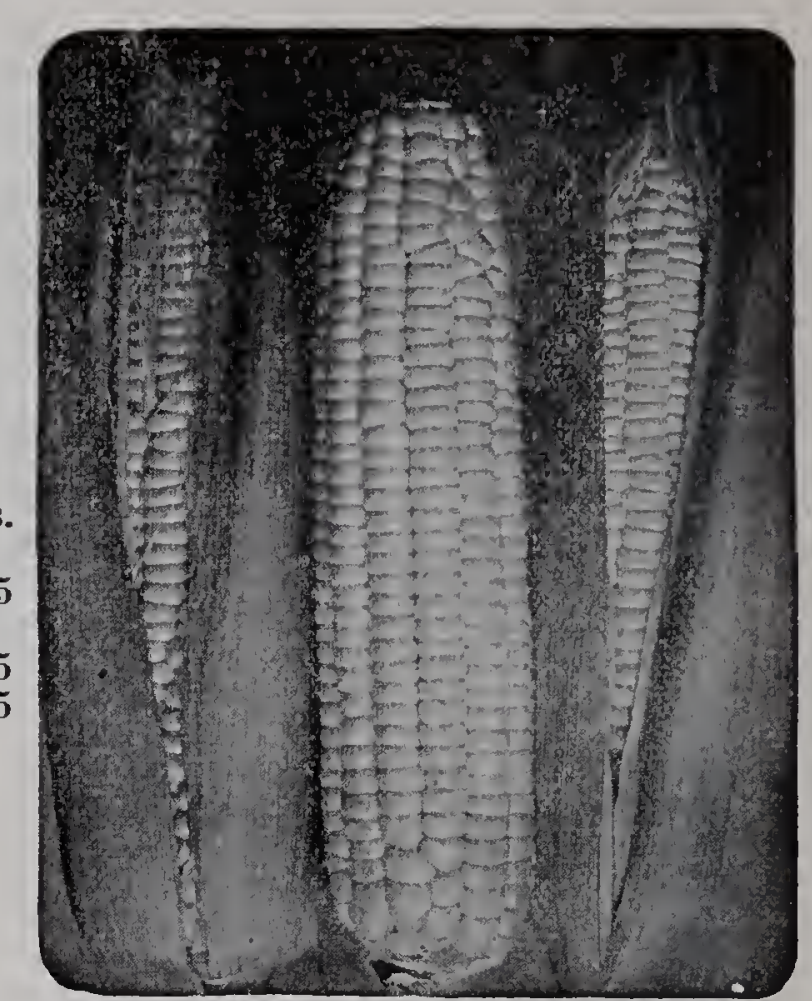

Stowell's Evergreen Sweet Corn

POP CORN L Japanese

Hulless $.15 \quad .25$

Queen's

Golden $\quad .15 \quad .25$

White Rice .15 .25

Wholesale and Retail Pop Corn for Popping Purposes - Write f o r

Prices.

SWEET or SUGAR CORN Pkt. 1/2 Lb. 1 Lb. 2 Lbs.

Peep $O^{\prime}$ Day ..................... .10

Golden Bantam ................ .10

Golden Sunshine ................15

Black Mexican ....10

Early Minnesota ............... .10

Evergreen ....................... 10

Country Gentleman ......... 10

White Corey ...................... 10

Whipple's Early White. .10

CUCUMBER Pkt.

Green Cluster.................. .05

White Spine .................... 05

Early Frame ...................... .05

Chicago Pickling ......... 05

Davis Perfect .................. .05

Long Green ...................... .05

Thornburn's Everbearing .05
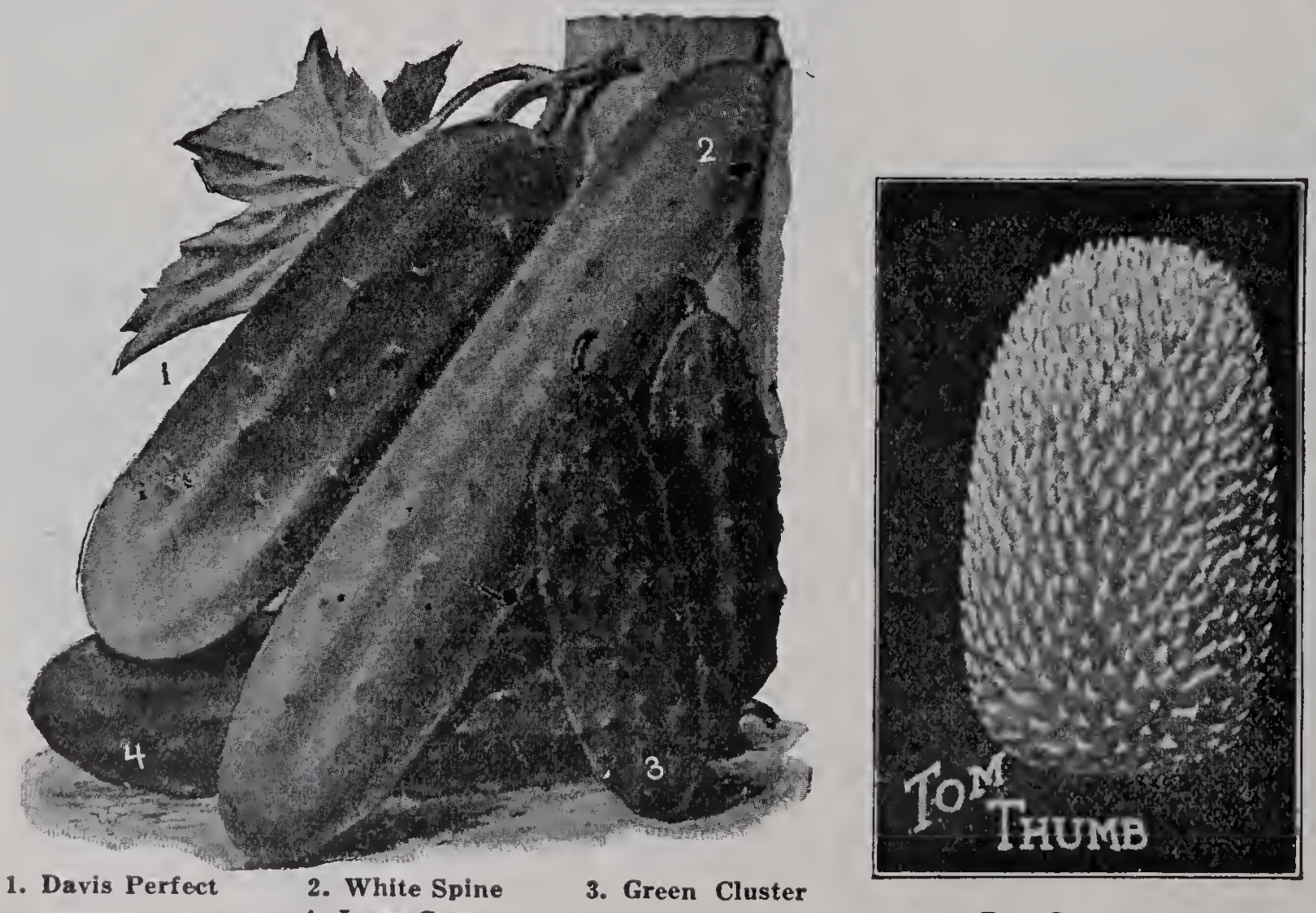

$\begin{array}{lll}.15 & .25 & .45\end{array}$

$.15 \quad .25 \quad .45$

$\begin{array}{lll}.20 & .30 & .55\end{array}$

$.15 \quad .25 \quad .45$

$.15 \quad .25$

$.15 \quad .25$

$.15 \quad .25$

$.15 \quad .25$

$.15 \quad .25$

$$
.45
$$

Oz. $\quad 1 / 2 \mathrm{Lb} .1 \mathrm{Lb}$.

$\begin{array}{lll}.10 & .55 & 1.00\end{array}$

$\begin{array}{lll}.10 & .55 & 1.00\end{array}$

$\begin{array}{lll}.10 & .55 & 1.00\end{array}$

$\begin{array}{lll}.10 & .55 & 1.00\end{array}$

$\begin{array}{lll}.10 & .55 & 1.00 \\ .10 & .55 & 1.00\end{array}$

$\begin{array}{lll}.10 & .55 & 1.00\end{array}$

$\begin{array}{lll}.10 & .55 & 1.00 \\ .10 & .55 & 1.00\end{array}$

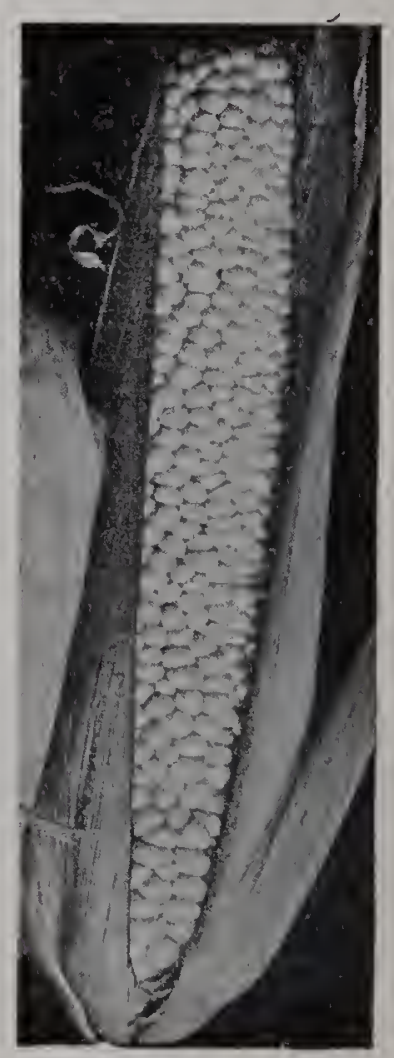

Pop Corn

Country Gentleman 

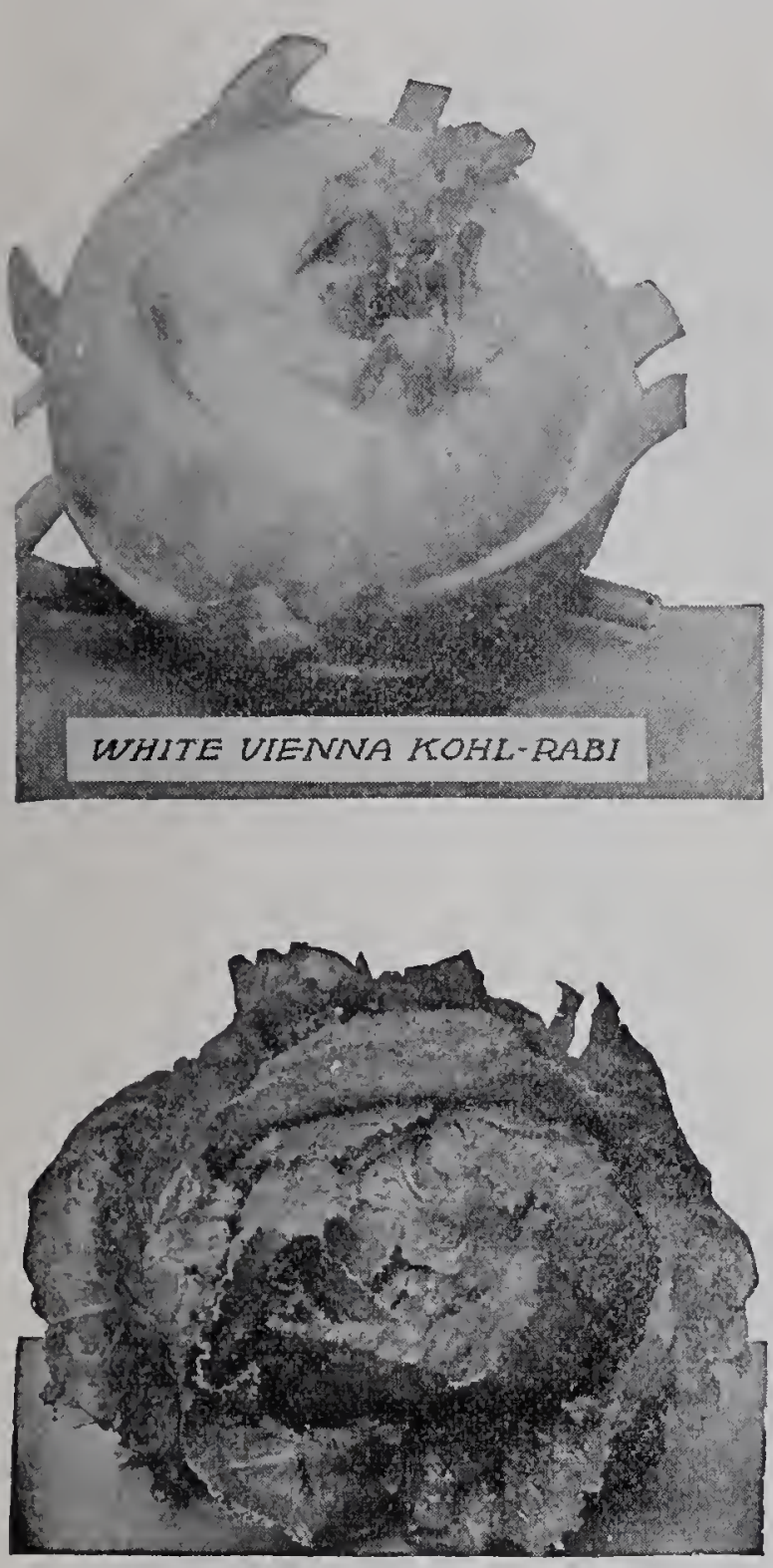

New York Head Lettuce

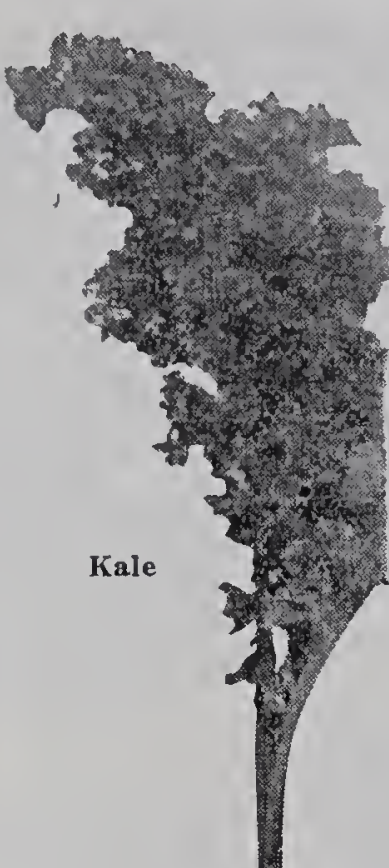

Pkt. Oz.

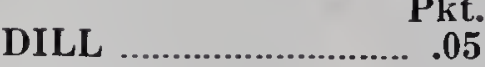

ENDIVE

Green Curled

.05

White Curled

.05

EGG PLANT

Early Long Purple .10

Black Beauty .......... .10

Imp. N. Y. Purple.. .10

KALE

Dwarf German ...... .05

Tall Green Curled.. .05

KOHL RABI

Purple Vienna ........ .05

White Vienna.

LEEK

Large Careton........ .05
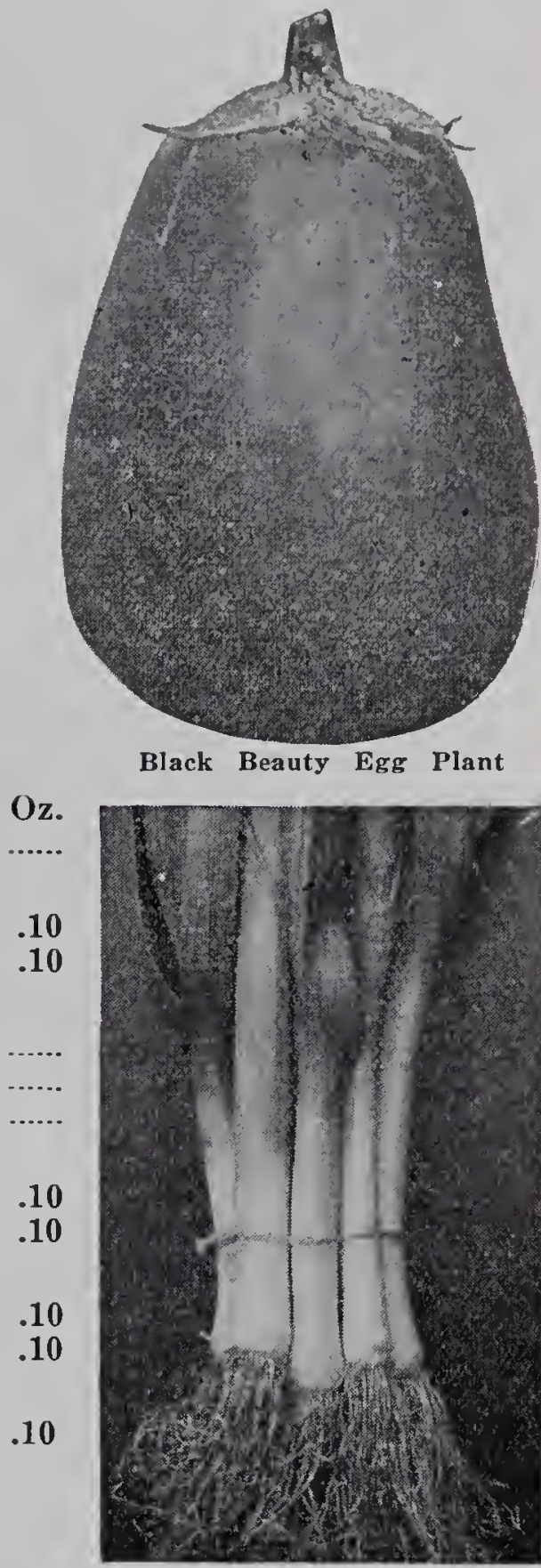

Pkt. Oz.
LETTUCE, Leaved

Early Curled Simpson

Black Seeded Simpson

Grand Rapids

Prizehead

.05

.05

.05

\section{HEAD LETTUCE}

Hanson

Boston Market

New York

Big Boston

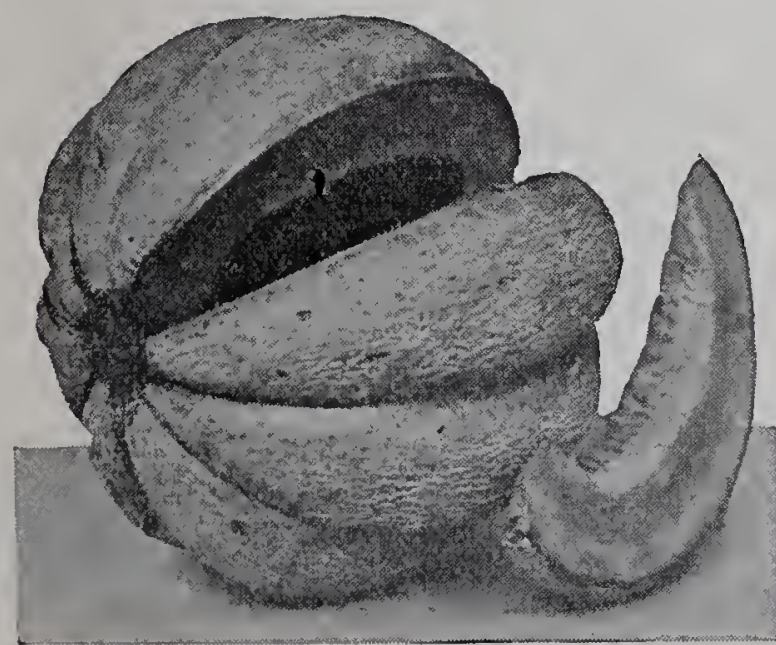

Emerald Gem Muskmelons
.10
.10
.10
.10

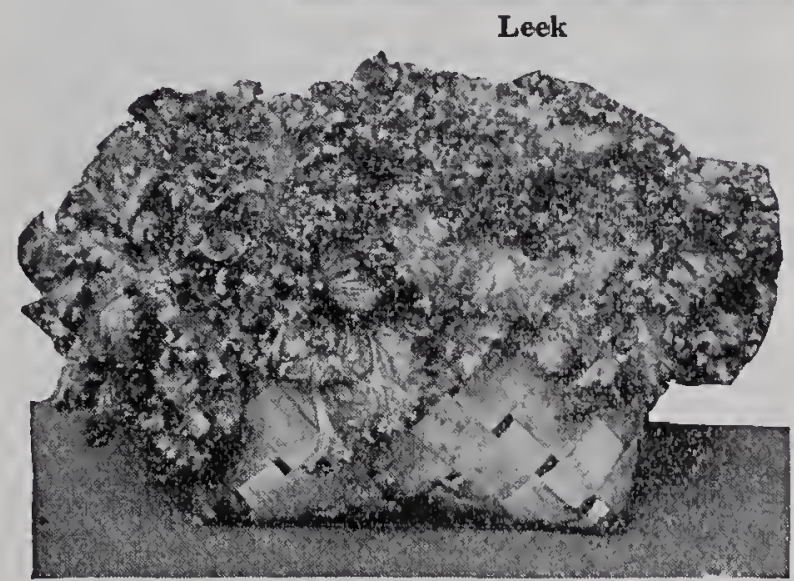

Grand Rapids Lettuce

Pkt. Oz.

Hearts of Gold............................................. .05 $\quad .10$

Netted Gem ................ .05

Honey Dew ......................................................

Rocky Ford ....................................................

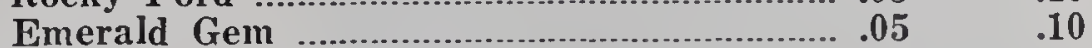

Barnard's Golden Hearted.............................. .05 $\quad .05$

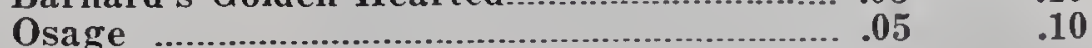

Hackensack

Banana .....................................................................

Improved Large Nutmeg

Lake Champlain ................................................ .05

Milwaukee Market ................. $05 \quad .10$ 


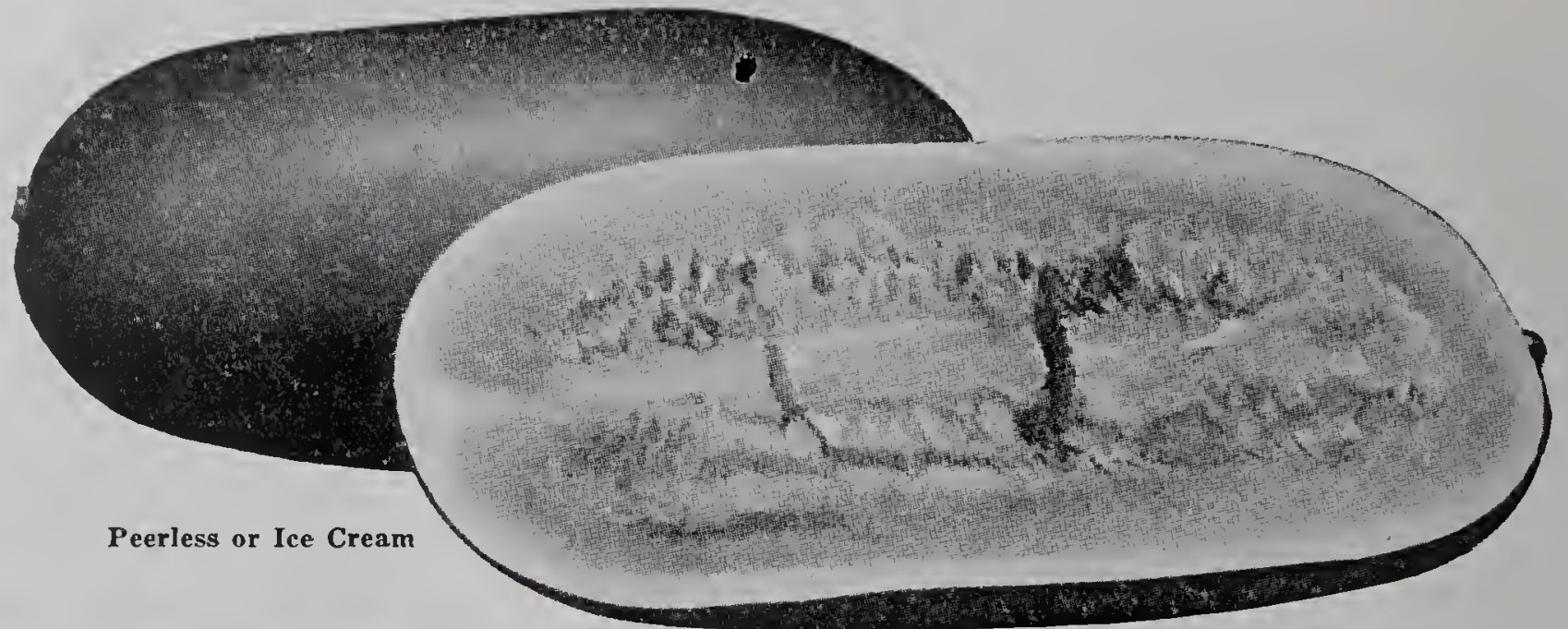

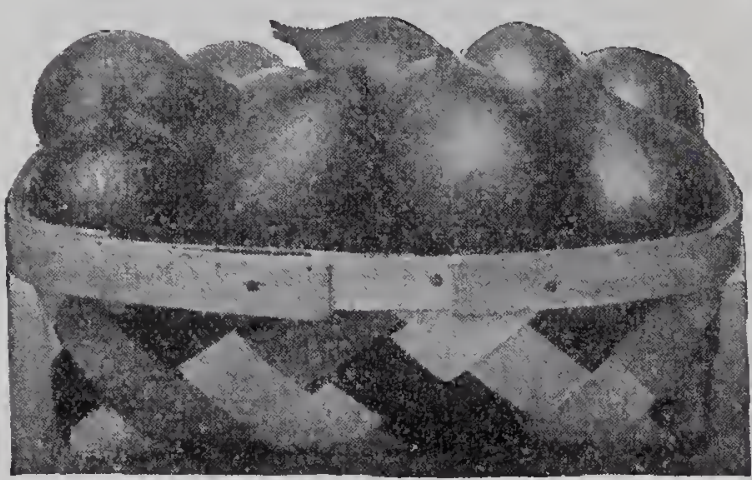

Southport Red Globe

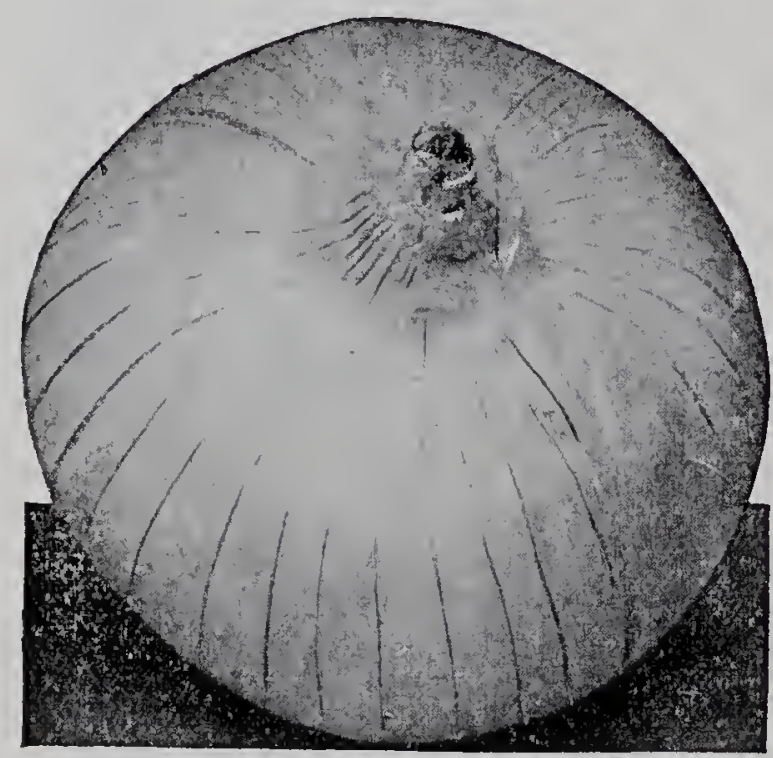

White Portugal Onion

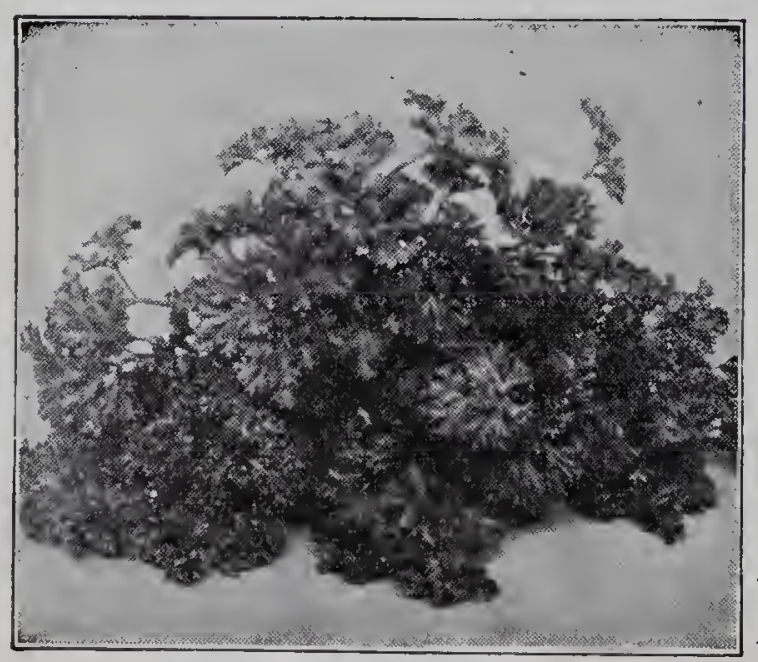

Champion Moss Curled Parsley

\section{WATERMELON}

\begin{tabular}{|c|c|c|}
\hline & Pkt. & $\mathrm{Oz}$. \\
\hline Phinney's Early & .05 & .10 \\
\hline Peerless or Ice Cream. & .05 & .10 \\
\hline 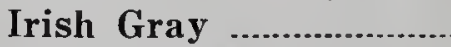 & .05 & .10 \\
\hline Halbert Honey .............. & .05 & .10 \\
\hline Tom Watson ..... & .05 & .10 \\
\hline Monte Cristo & .05 & .10 \\
\hline Sweetheart .... & .05 & .10 \\
\hline Citron ............... & .05 & .10 \\
\hline
\end{tabular}

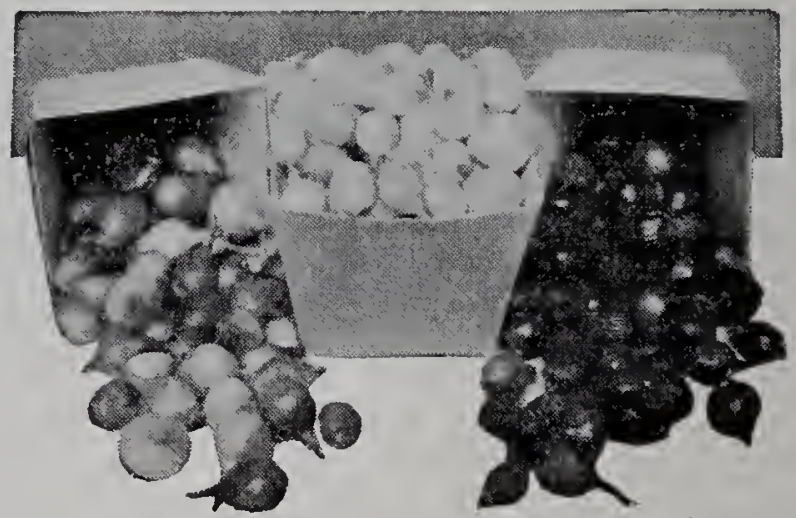

ONIONS

White Portugal

Prizetaker, Yellow Skin .05 $\quad .15 \quad 1.15 \quad 2.25$

Yellow Globe Danvers.. $.05 \quad .15 \quad 1.15 \quad 2.25$

Southport Yellow Globe $.05 \quad .15 \quad 1.15 \quad 2.25$

Southport Red Globe.... $.05 \quad .15 \quad 1.15 \quad 2.25$

Red Wethersfield ........... $05 \quad .15 \quad 1.15 \quad 2.25$

ONION PLANTS Write for prices

ONION SETS $1 \mathrm{Lb} .2 \mathrm{Lbs}$

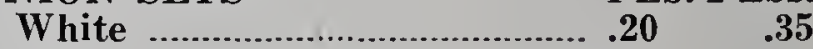

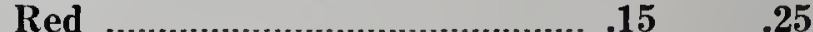

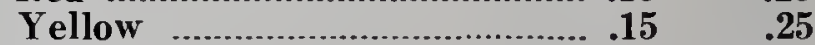

PARSNIP $\quad$ Pkt. Oz.

Hollow Crown ........................... .05 $\quad .10$

Market Garden .......................... .05 .10

PARSLEY

Champion Moss Curled.............. .05 .10

PEANUTS $1 / 2$ Lb. $1 \mathrm{Lb}$.

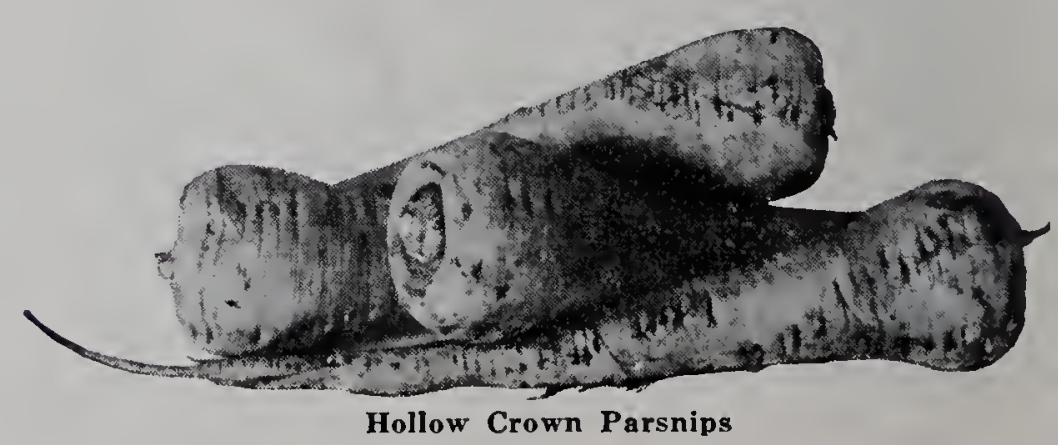




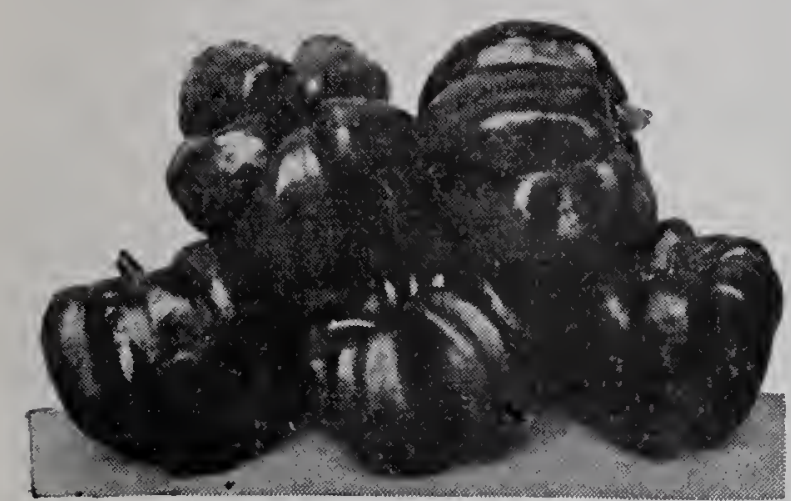

PEPPERS
Sweet Mountain (mild) $\quad . . . . .10$

Large Bell (hot)....................10

Red Cayenne ............................ 10

Pimento (very mild) ............ 10

Ruby King

.10

PEPPERS ARE INDISPENSABLE

FOR SEASONING SOUPS AND

MEATS; ALSO FOR PICKLING

Ruby King Peppers

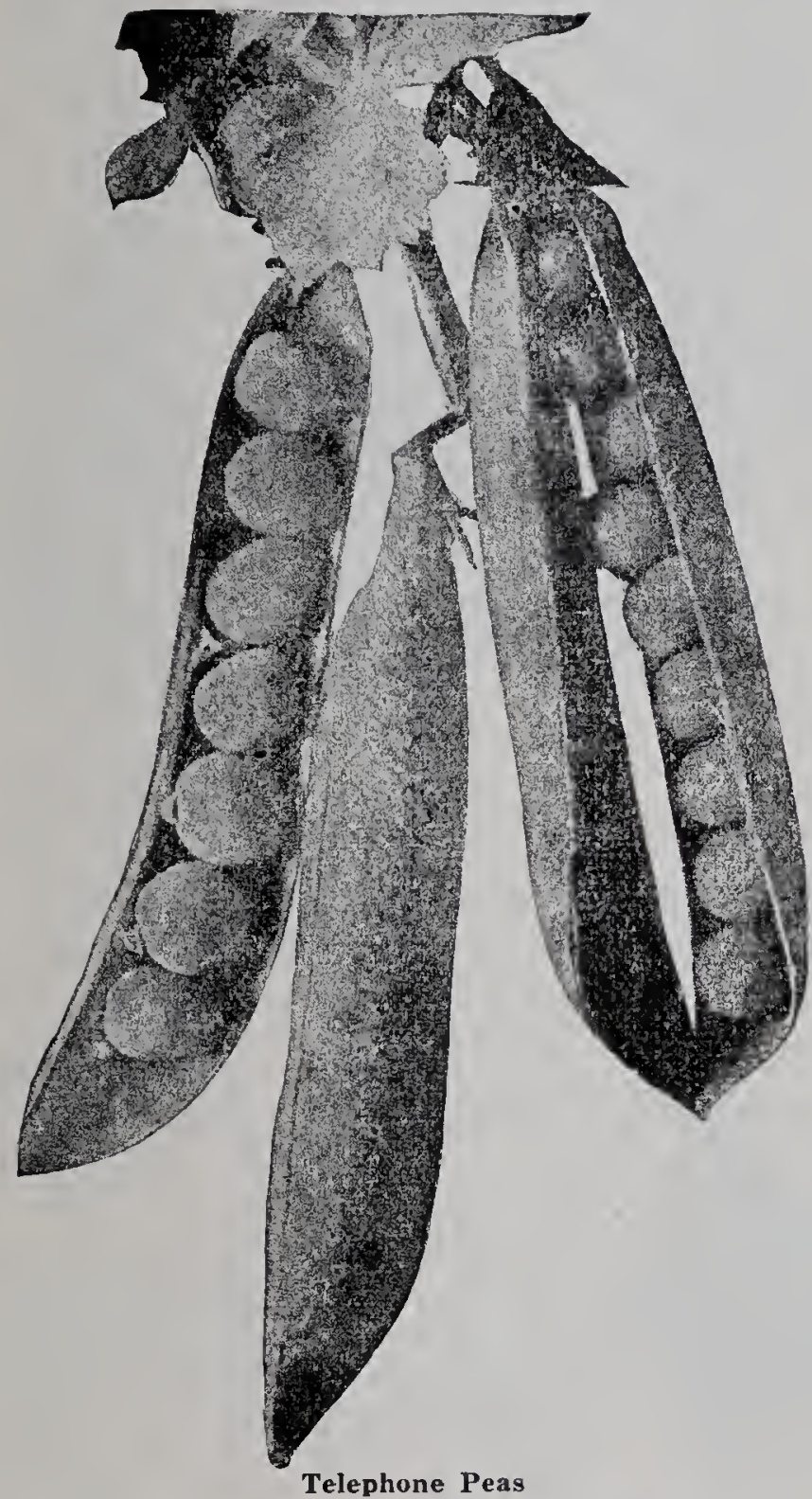

\begin{tabular}{c}
\hline PEAS \\
DESERVE \\
A PLACE \\
IN EVERY \\
HOME \\
GARDEN \\
PLANT \\
ROCHELLE \\
QUALITY \\
SEEDS \\
\hline \hline
\end{tabular}

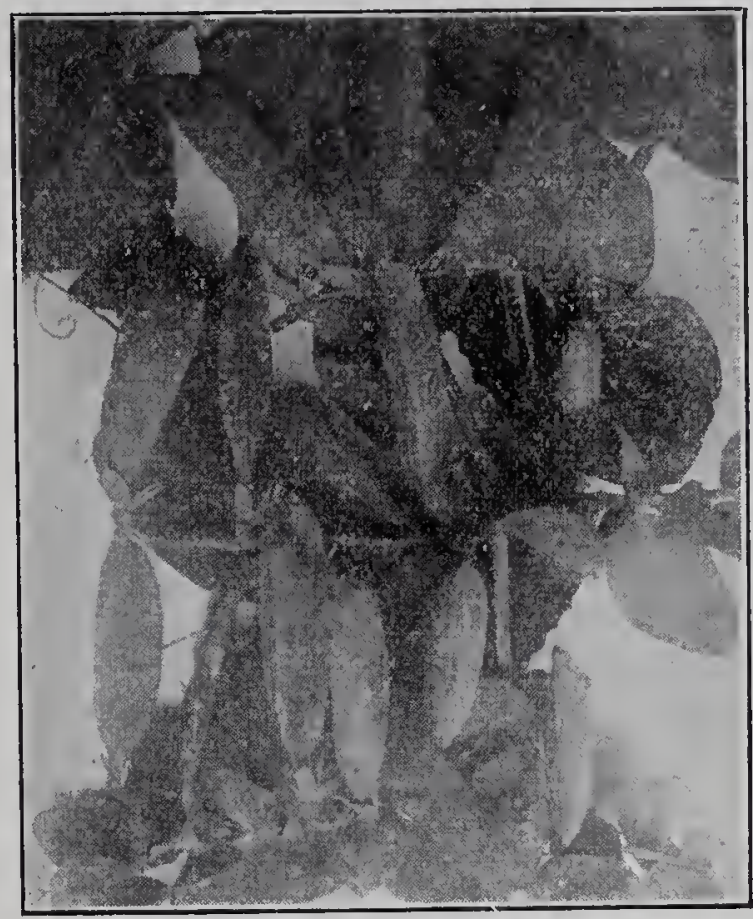

Peas, Premium Gem

PEAS (Smooth Varieties) Pkt. 1/2 Lb. 1 Lb. 2 Lbs.

First and Best (early).......... .10

Alaska (early) ......................10

(Wrinkled Varieties-Dwarf)

Premium Gem (early)......... .10

Little Marvel (early)............. 10

Daisy-Dwarf Telephone...... .10

American Wonder (early) .... .10

Nott's Excelsior (early).......10

(Half Dwarf Wrinkled Varieties)

Thomas Laxton ....................... .10

Gradus ...................................... 10

Potlateh ................................... .10

(Tall Growing Wrinkled Varieties)

Champion of England............ .10

Telephone .............................. .10

(Tall Smooth Varieties)

Large White Eye Marrowfat .10

Large Black Eye Marrowfat .10

Dwarf Gray Sugar.

.10

$\begin{array}{lll}.20 & .40 & .75 \\ .20 & .40 & .75 \\ .20 & .40 & .75 \\ .20 & .40 & .75 \\ .20 & .40 & .75 \\ .20 & .40 & .75 \\ .20 & .40 & .75 \\ .20 & .40 & .75 \\ .20 & .40 & .75 \\ .20 & .40 & .75 \\ .20 & .40 & .75 \\ .20 & .40 & .75 \\ & & \\ .20 & .40 & .75 \\ .20 & .40 & .75 \\ .20 & .40 & .75\end{array}$

PUMPKINS

Pkt.

Japanese Pie

.05

Small Sweet or Sugar............ .05

Large Yellow or Conn. Field .05

Giant Mammoth (yellow).... .05

Mammoth Tours
Oz. 1/2 Lb. $1 \mathrm{Lb}$.

$\begin{array}{lll}.10 & .55 & 1.00\end{array}$

$\begin{array}{lll}.10 & .45 & .85\end{array}$

$\begin{array}{lll}.10 & .45 & .85\end{array}$

$\begin{array}{lll}.10 & .65 & 1.25\end{array}$

$\begin{array}{lll}.10 & .55 & 1.00\end{array}$ 


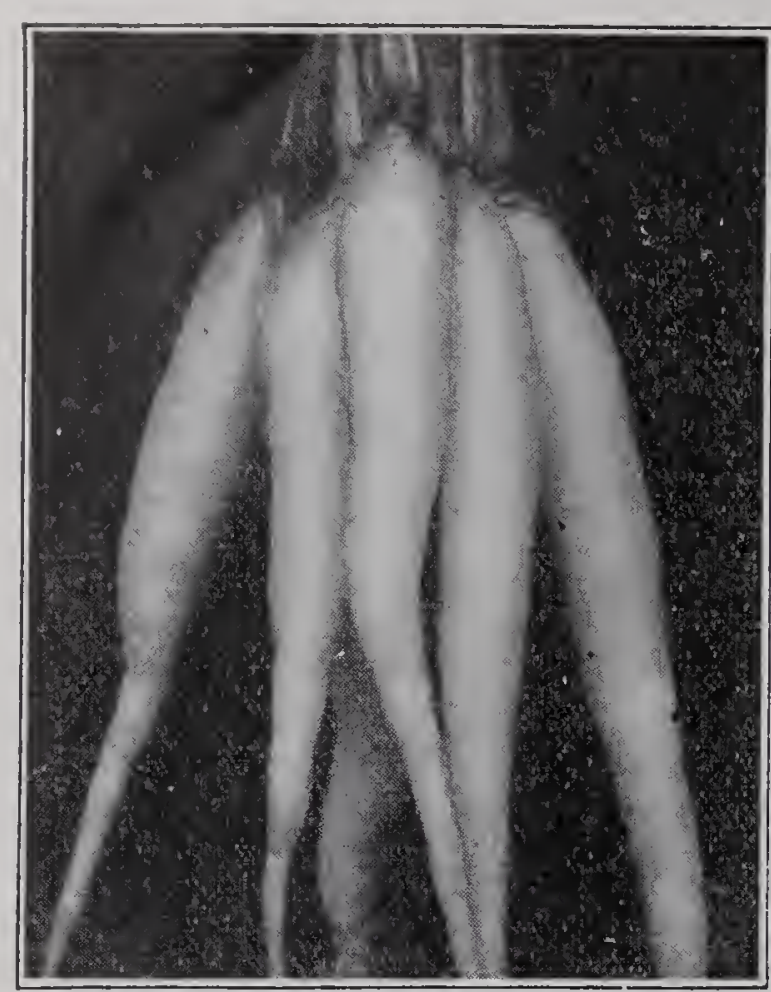

Lady Finger Radishes

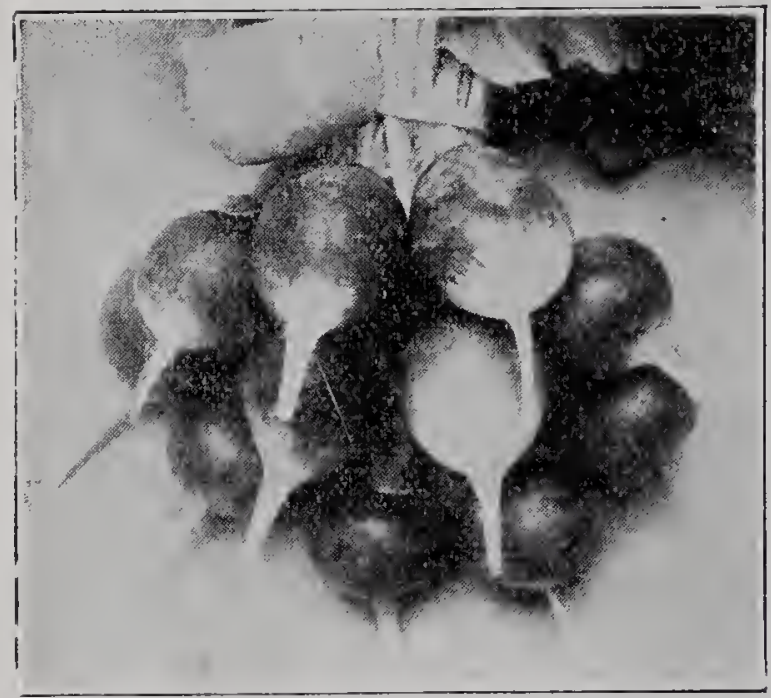

French Breakfast Radishes

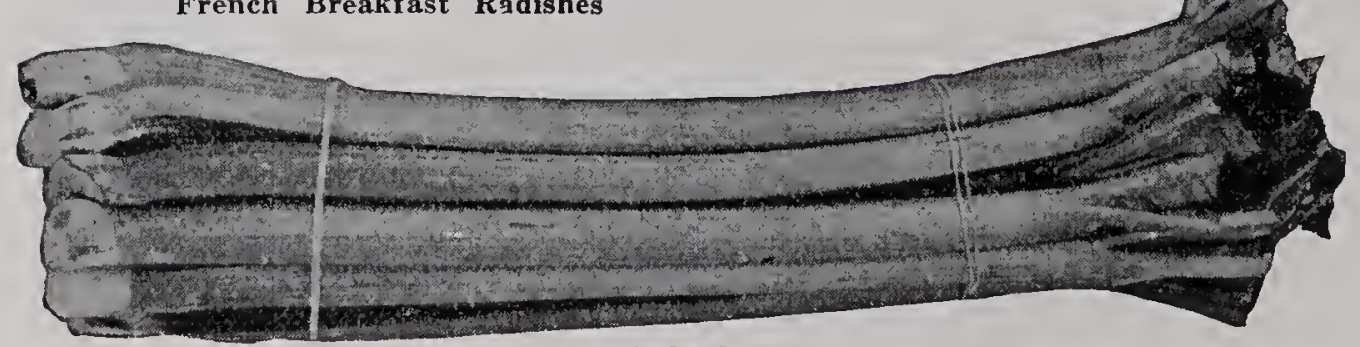

Rhubarb

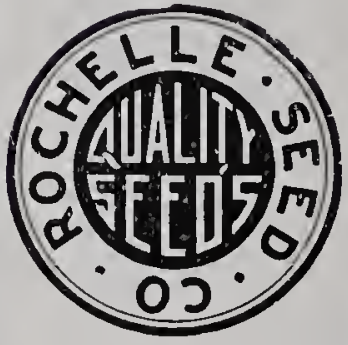

RHUBARB

Pkt. Oz.

SALSIFY

Mammoth Sandwich Island. .05

.15

SPINACH

Pkt. Oz.

New Zealand............. .05 .10 Thick Leaved ........... .05 $\quad .10$

SQUASH

Table Queen............. .10 $\quad .20$

Hubbard ................... .05 $\quad .05$

Golden Hubbard...... .05 .10

Crookneck, summer .05 .10

White Bush.............. .05 $\quad .10$

Golden Custard ...... .05 .10

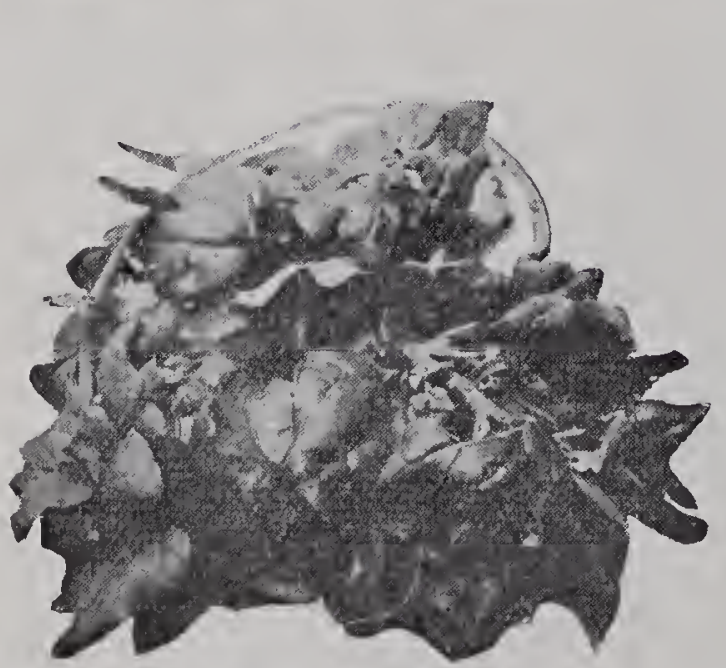

Thick Leaved Spinach

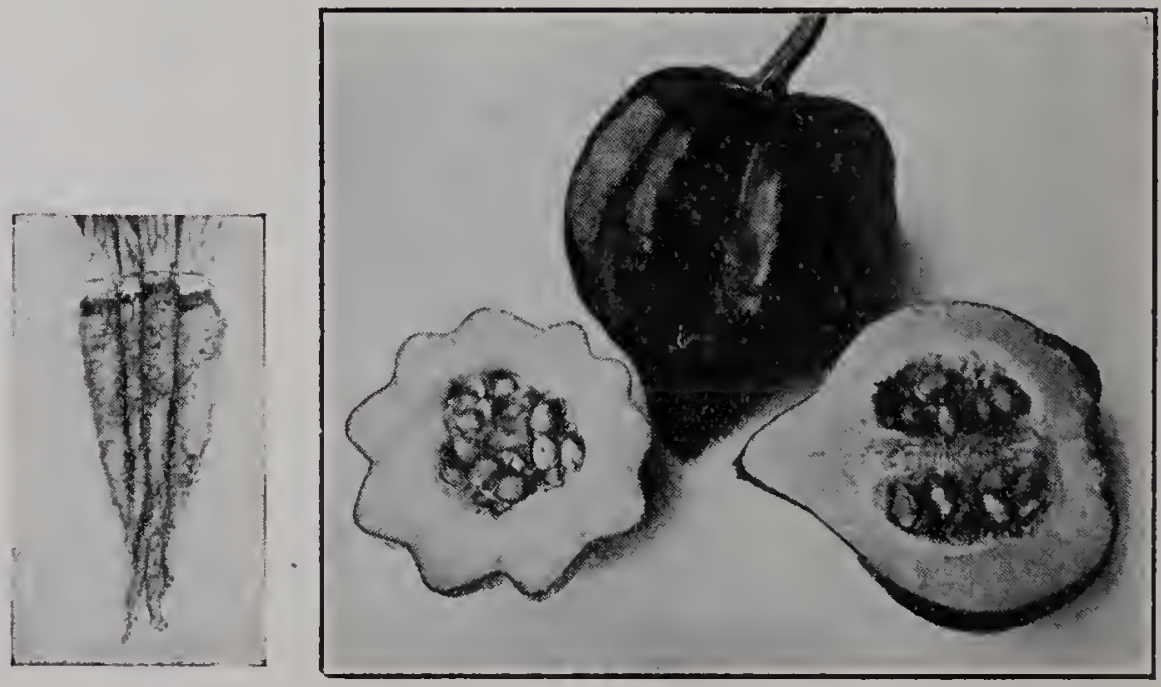

Salsify 


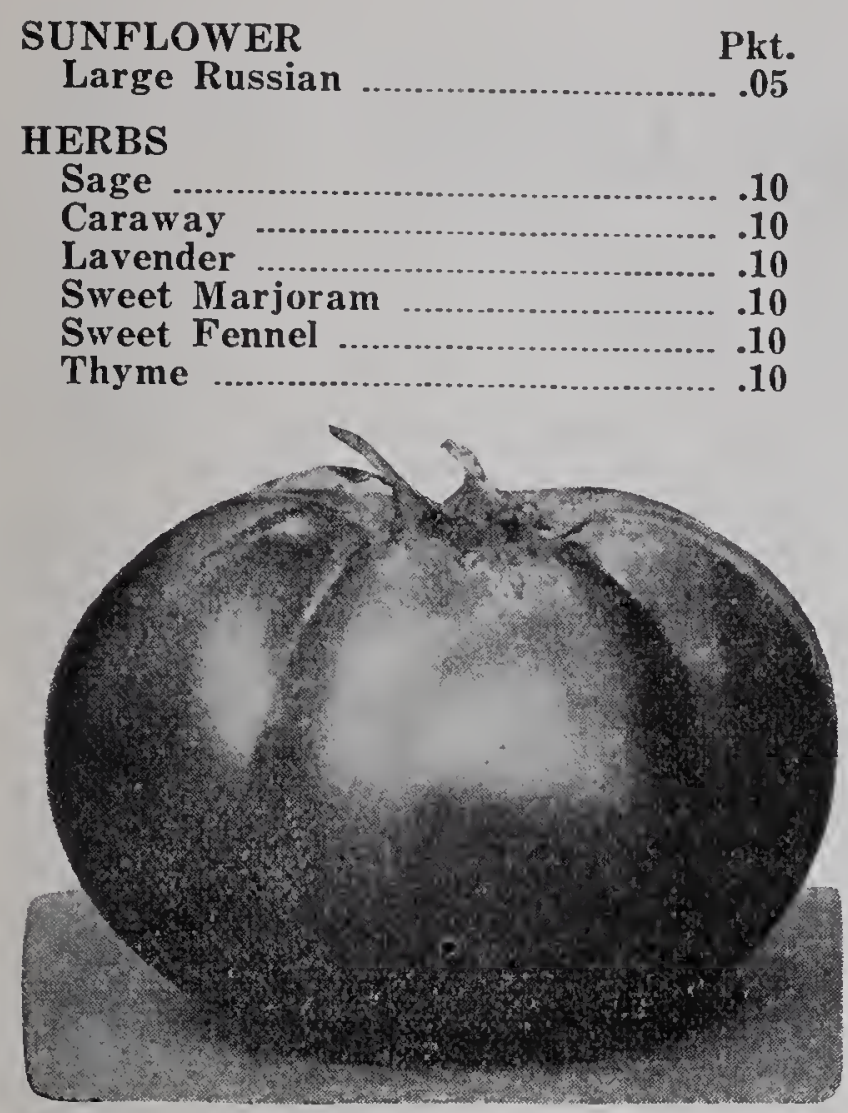

Beefsteak Tomato

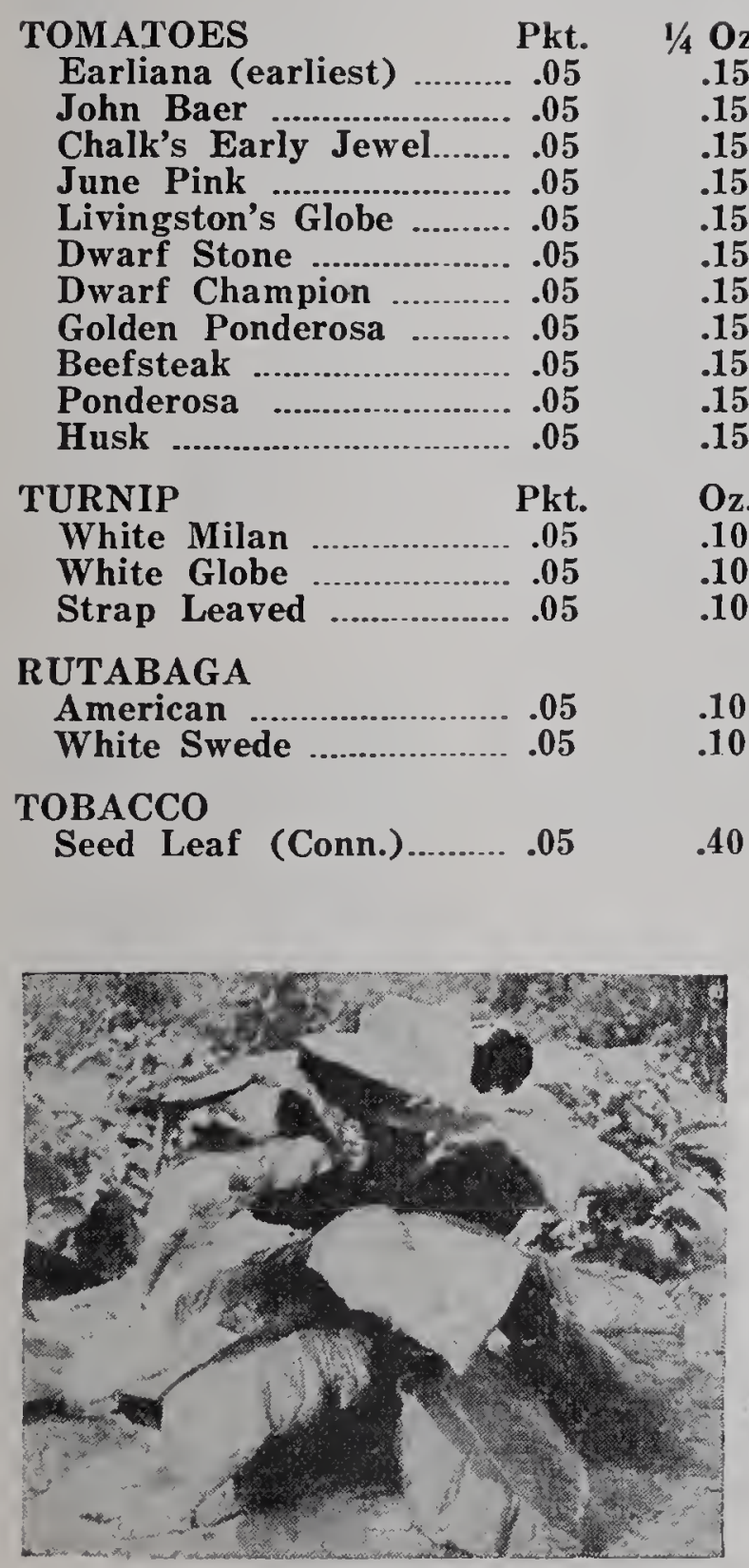

Tobacco

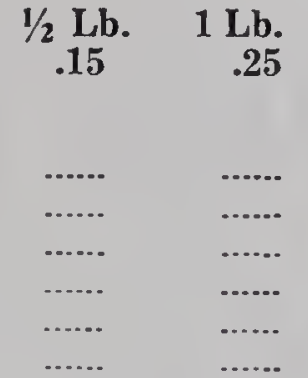

\section{REMEMBER}

You want lots of tomatoes for slicing, cooking and canning.
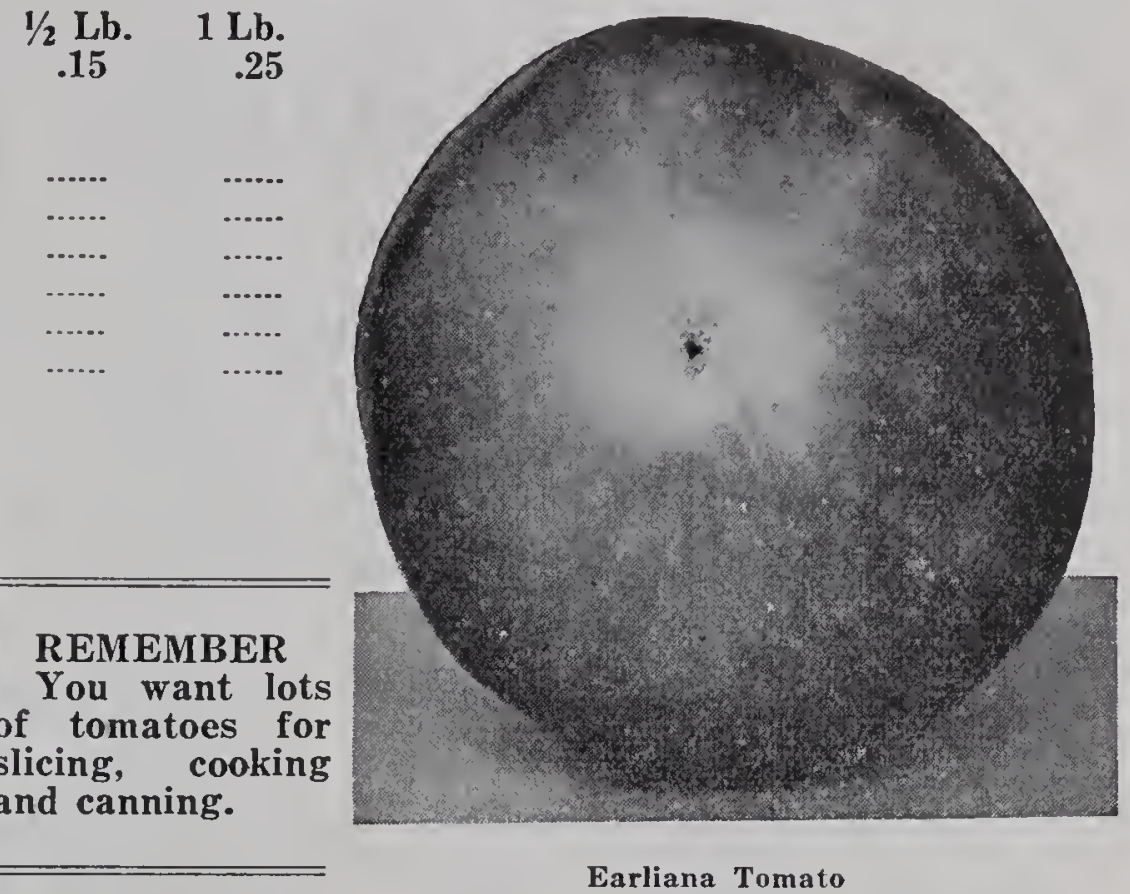

Earliana Tomato

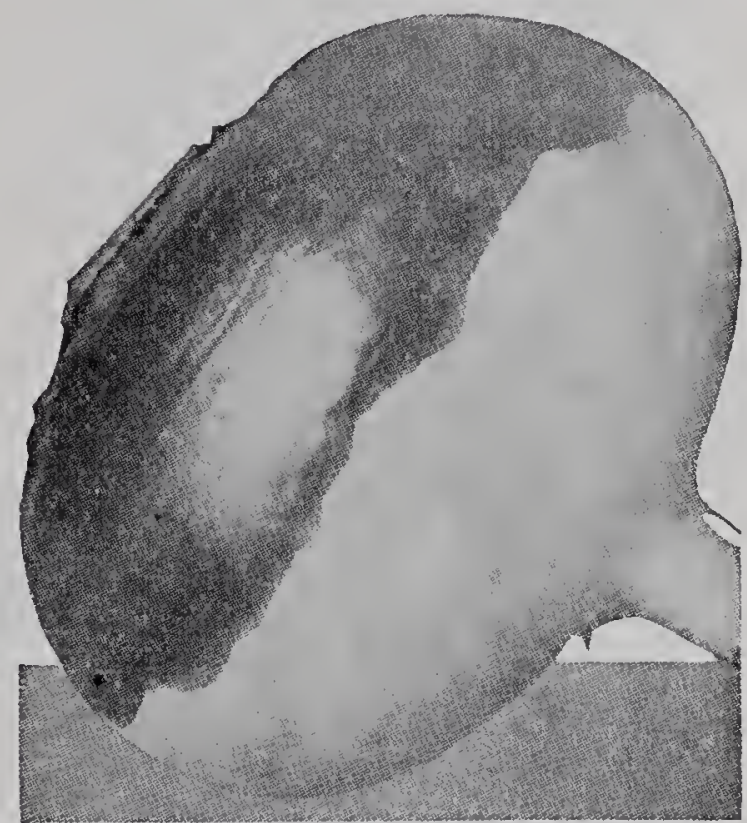

White Globe Turnip

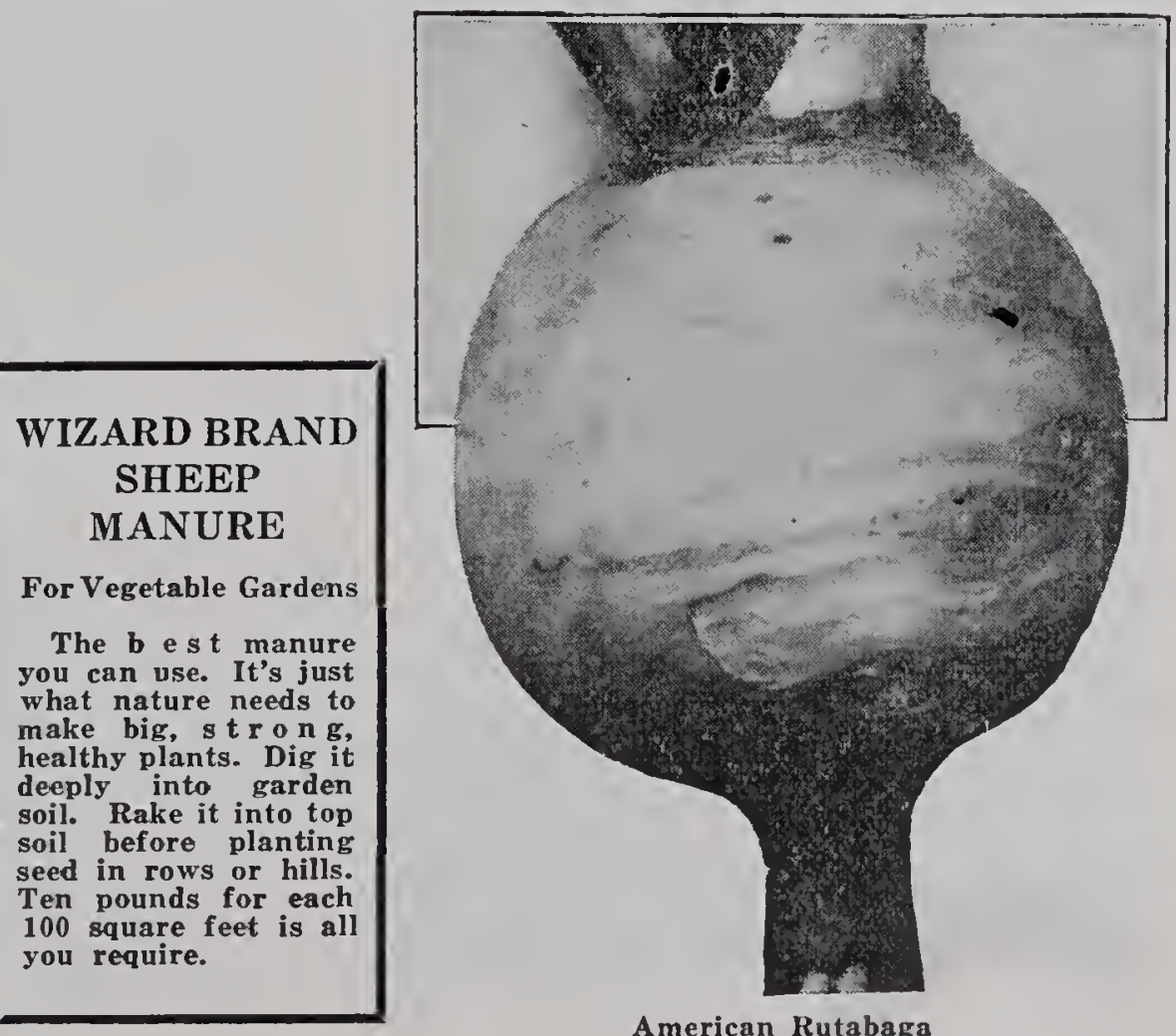

American Rutabaga 


\section{FLOWER SEEDS}

\section{Everybody Loves Flowers}

Flowers are inexpensive. If you select the right kind, they are as easy to grow as weeds, but everyone is not familiar with the kind of flower seed to buy to make the quickest and most satisfactory showing. We are pleased, however, to offer a very choice list of the most popular and easily grown flower seeds. They are strictly fresh, new crop and the very best obtainable.

ANNUALS are those flowers that bloom and ripen their seed the first year and then die. Among these are the asters, balsams, mignonette, sweet peas, nasturtiums, morning glories, and many other very brilliant and fragrant varieties.

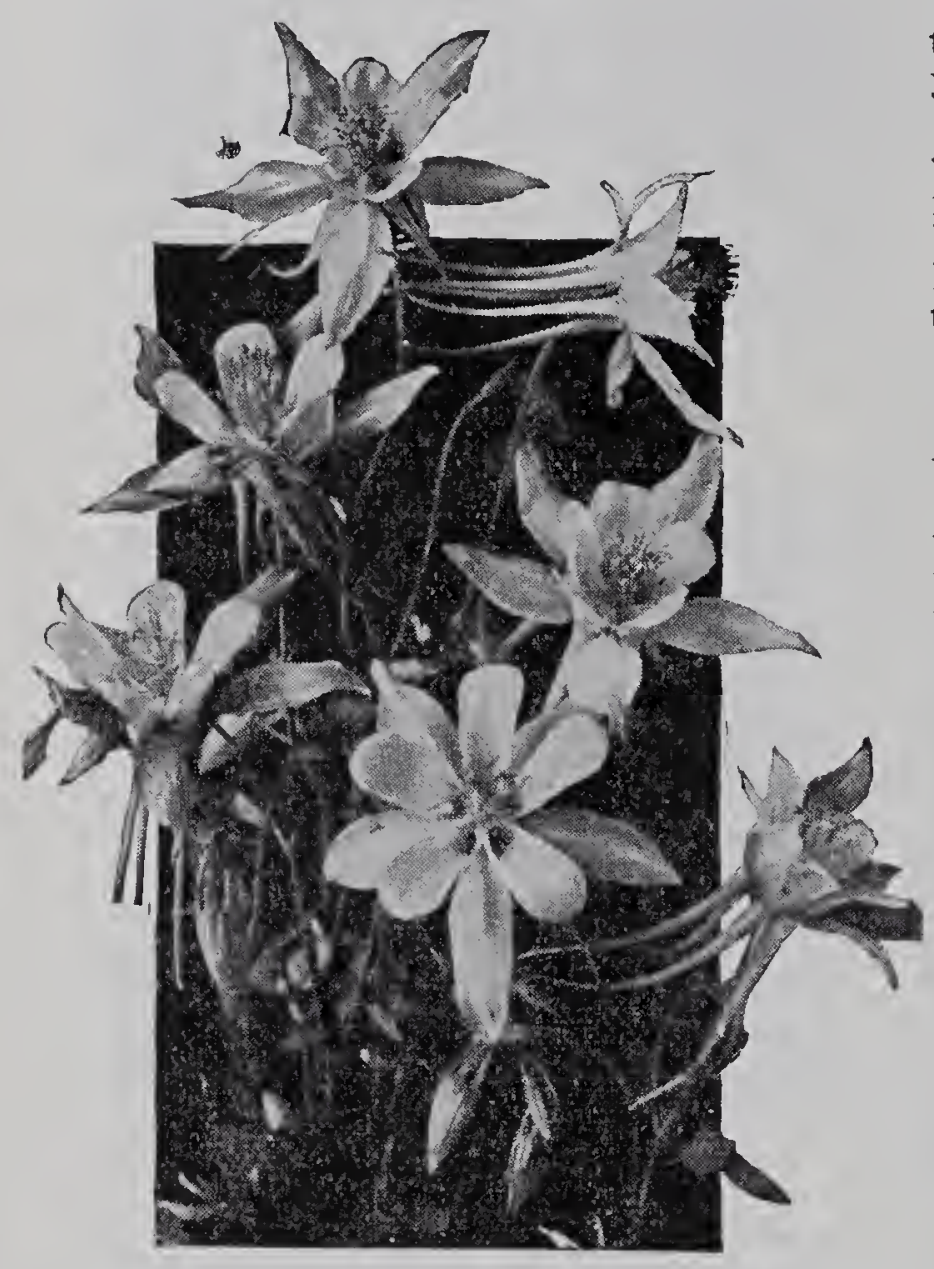

Long Spurred Columbine (Aquilegia)

BIENNIALS from seed generally bloom the second year, then die. Some varieties, if planted early, bloom the first year; therefore are treated as annuals.

PERENNIALS are those that endure our long winters with little or no protection and live and bloom several years in succession. A large majority of the perennials will bloom the first year if started early in the spring. Hollyhocks, columbine, larkspur, foxglove, poppies, etc., are in this class.

\section{ALL FLOWERS SENT POSTPAID UPON RECEIPT OF PRICE}

AGERATUM

Imperial Dwarf Blue.

Pkt.

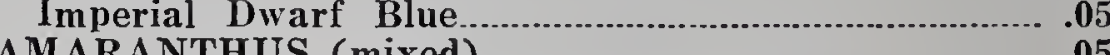

ANTRARHUS (mixed) ……...................................... .05

ANTIRRHINUM-Snapdragon (mixed) ...................... .05

ALYSSUM

Little Gem ……................................................................ 05

AQUILEGrA-Columbine

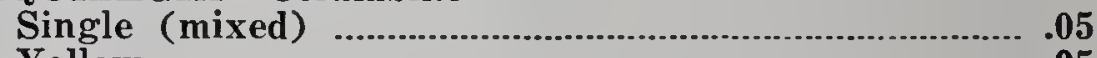

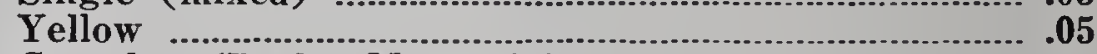

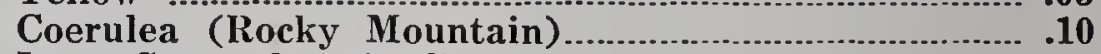

Long Spurred (mixed) ........................................................ .15

ASTERS

Hohenzollern (mixed) …............................................... .10

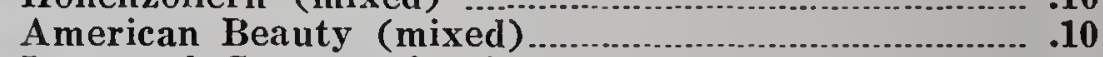

Improved Crego (mixed) ........................................................ .10

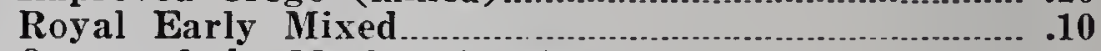

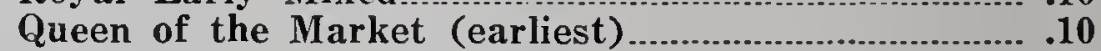

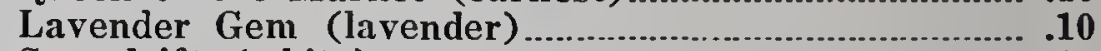

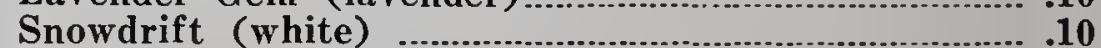

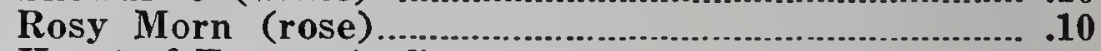

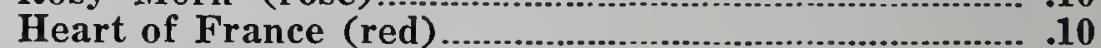

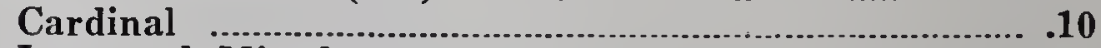

Improved Mixed …….............................................................

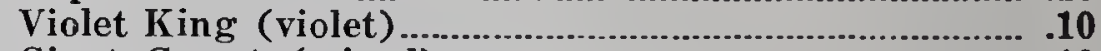

Giant Comet (mixed) ……….................................................... .10

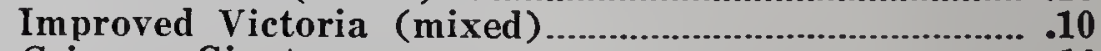

Crimson Giant ....................................................................

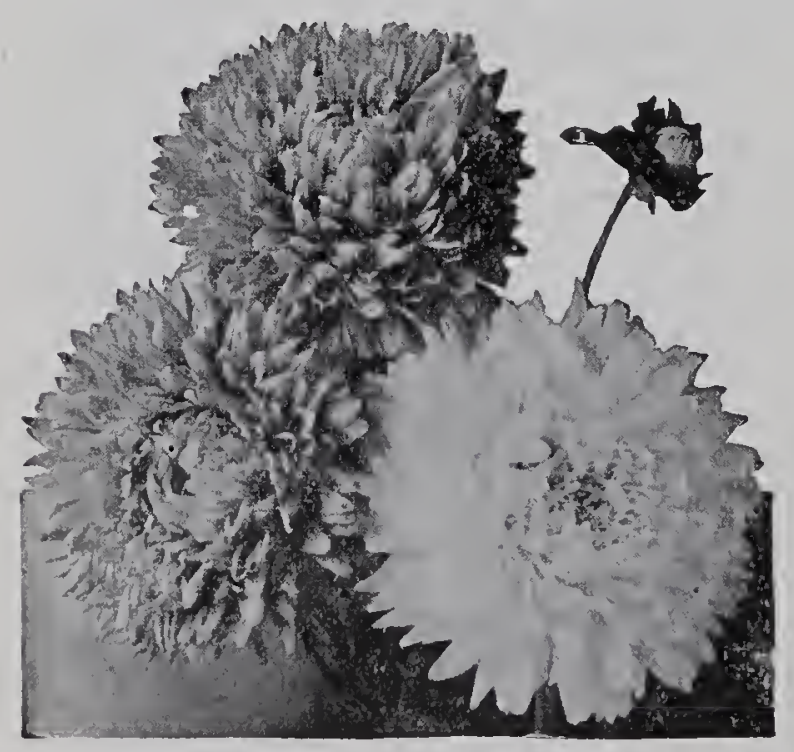

Hohenzollern Asters, Mixed

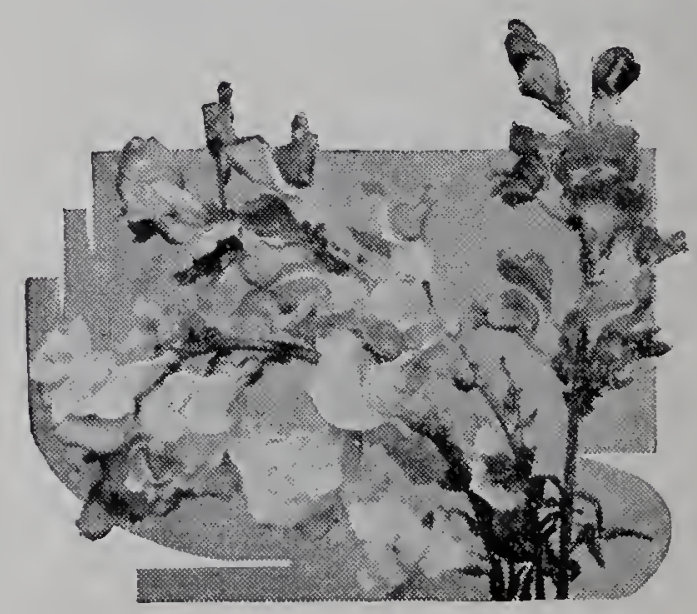

Snapdragons 


\section{BALSAM}

Camelia Flowered (double)

BELLIS PERENNIS

BEANS, SCARLET RUNNER

BALLOON VINE (Annual).

CANNA

CENTAUREA-GYMNOCARPA (Dusty Miller).... .05

CELOSIA-COCK SCOMB

Thompsoni

.05

Empress

President Theirs

Dwarf Mixed

CHINESE WOOLFLOWER

C.AI,ENDULA (Orange King)

CALLIOPSIS

CANDYTUFT

Giant Hyacinth Flowered

CLARKIA

Double Mixed

Pkt.

.05

.05

\section{5} 05 5 .05

.05

.05

.05

.05

.05

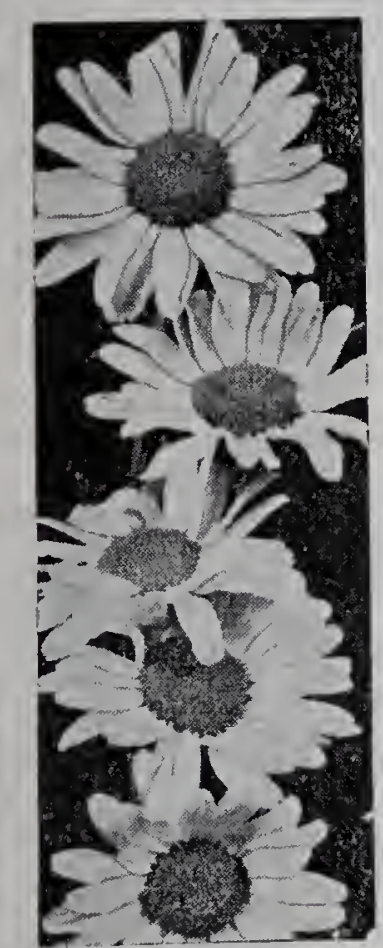

Shasta Daisies

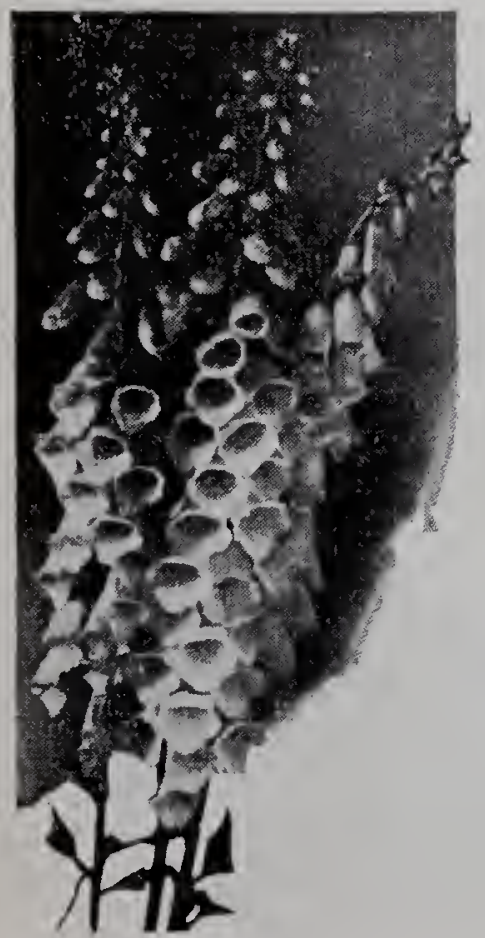

Digitalis or Fox Glove
COBAEA SCANDENS (Climber) ........................... .05
CHRYSANTHEM CARNATION

Marguerite

Red Grenadine

White

CARDINAL CLIMBER (Rapid Grower)

CYPRESS VINE (Tender Annual)

CANARY BIRD VINE (Yellow Flowers)

COSMOS-Dwarf

Mammoth

Double Crested

COREOPSIS

Lanceolata (yellow)

CAMPANULA-CANTERBURY BELL

Cup and Saucer.

Double or Single.

DELPHINIUM-Perennial

Belladonna

CASTOR BEANS

ESCHOLTZIA (California Poppy)

SINGLE DA HLIA

GLADIOLI BULBS, per dozen

GODETIA (Satin Flower)

GOMPHRENA-Everlasting

GOURDS

Dish Cloth

Sugar Trough

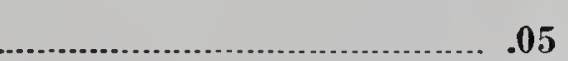

Bottle

Apple Shaped

Dipper ............................................................... 05

Hercules Club ............................................. 05

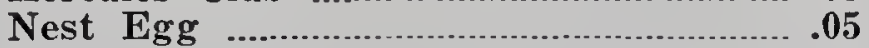

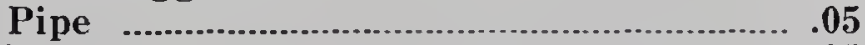

Pear

GYPSOPHILA-Baby Breath

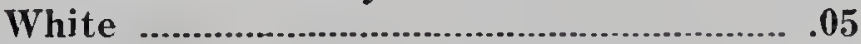

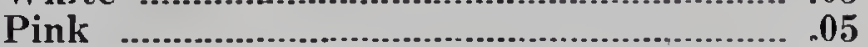

HELIOTROPE (Mixed Varieties)............... .05

HELICHRYSUM - Straw Flower ............... 05

HUMULUS JAPONICUS-Japanese Hops .05

HOLLYHOCKS-Double (Mixed) ............... .05

Single (mixed)

IPOMOEA

Moonvine Grandiflora

Setosia-Brazilian Moon Flower

Rubro Coerulea or Heavenly Blue........... .05

JAPANESE MORNING GLORY ................ .05

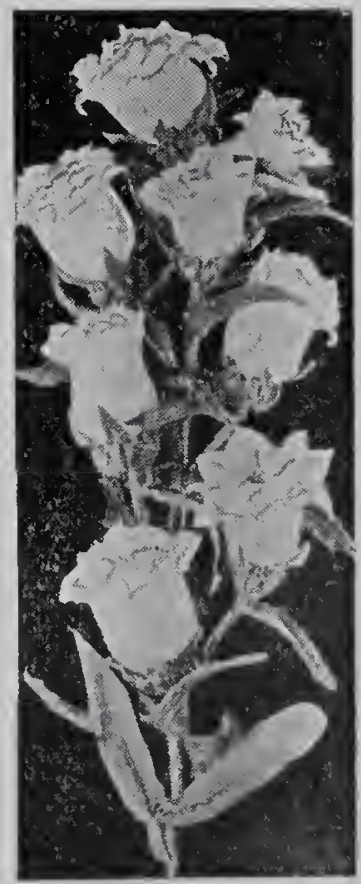

Campanula Double 


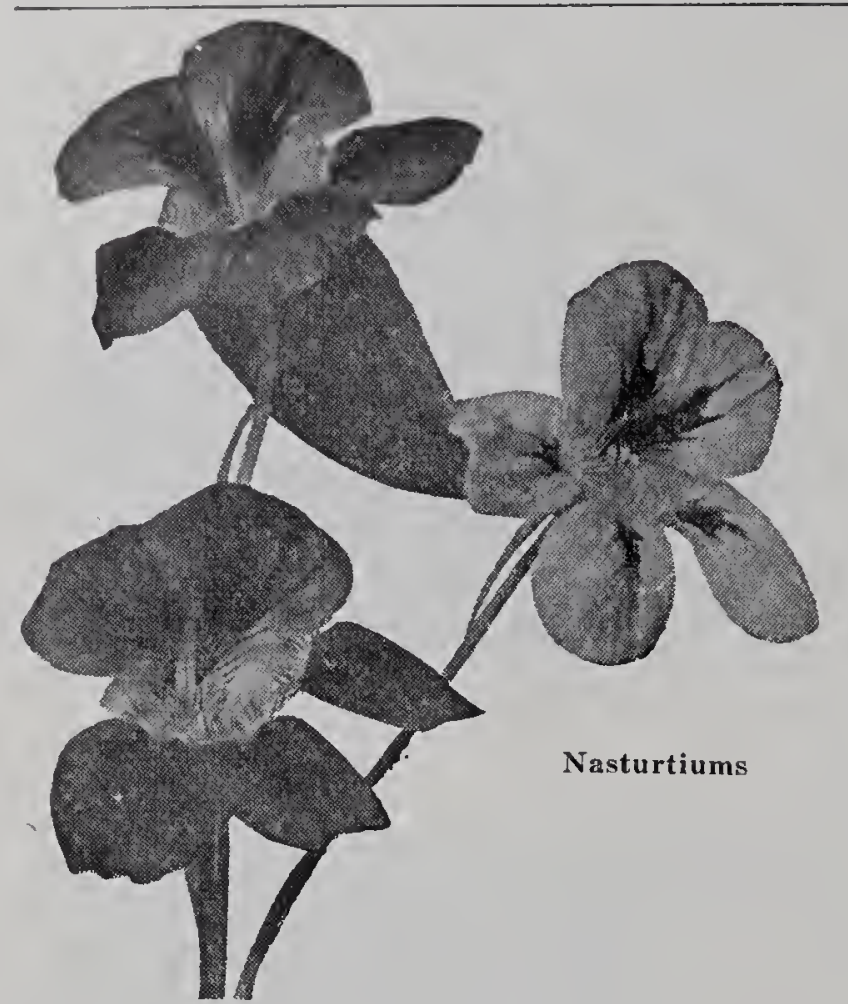

Pkt.

JOB'S TEARS

.05

LOBELIA

KUDZU VINE-(Perennial) ........................... 10

LUNARIA (Annual) ...... .10

LANTANIA

.05

LATHYRUS LATIFOLUYS

-Hardy Sweet Pea.. .10

MARIGOLD

Tall African

Dwarf French

Tagetes Signata Pum-

ila, Dwarf Yellow...... .05

LAVANDULA-English Lavender

.05

MIGNONETTE

NASTURTIUM

Dwarf

Tall

NICOTIANA

PANSTFS

Ideal Mixture

Prize Giant

Trimardeau

Masterpiece

.05 PENNISETUM

.05

PORTULACA-Moss Ros

Double

Single .10

LARKSPUR (Annual) Dark Blue ...................... .10

Branching Pink ............. .10

White ............................ 10

Carmine .......................... .10

Rocket ............................. 10

Hyacinth Flowered, Mixed

.05

\section{PETUNIAS}

The Rochelle Flower

Petunias are delightful in a sunny spot, developing into bushy plants completely covered with charming flowers in a most complete range and nearly all with a delicate fragrance.

Plant in rows or masses in the garden for brilliant effect. Seeds are very small and should be covered very lightly. Rosy Morn and Balcony Blue make a lovely combination for window boxes.

PHLOX

PINKS

Chinensis

Plumarius

POPPIES

PYRETHRUM (Perennial)

SCHIZANTHUS-But-

terfly Flower

SALVIA

Bonfire

SWEET WILLIAM
LUPINUS

PETUNIAS

SUMMER CYPRESS-Fire Bush.

Pkt.

TLOSSIS

SWEET PEA

Pkt. Oz.

Royal Mixed ........................................... .05

Spencer Mixed ............................................. .10 .15

SUNFLOWER-Double

SWEET SCABIOSA-Pin Cushion .................... .05

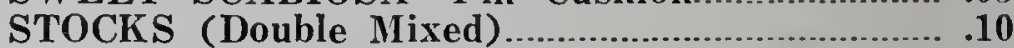

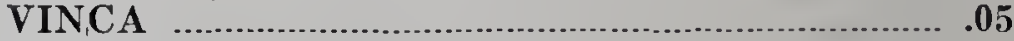

VERBENA Pkt.

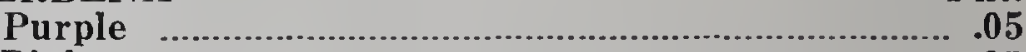

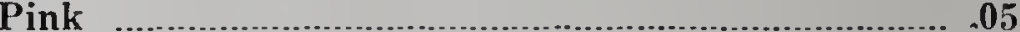

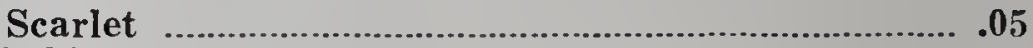

White

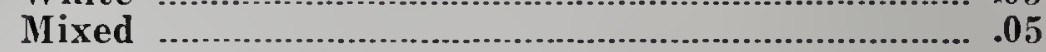

XERANTHEMUM-Everlasting ............................... .05

ZINNIA-Dahlia Flowered

Exquisite (Rose) ..................................................... .15

Golden State (Yellow)

Crimson Monarch (Red) ........................................... 15

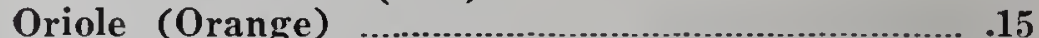

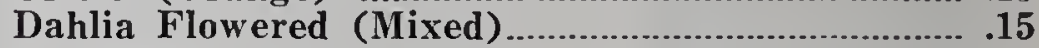

Red Riding Hood (Dwarf) ..................................... .10

Rosy Morn (Pink)........ .05 Balcony Blue (Indigo)...10

Single (mixed) ........... .05

Drummondi ….................. .05

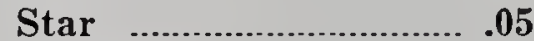

Carmine ........................ .05

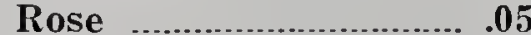

Mixed ............................. .05

.05

Heddewigi

Mixed ............................... .05

Shirley .......................... .05

Iceland (Perennial) .... .05

Oriental (Perennial) .... .05

Double Mixed ................ .05

Double Carnation ............ .05

.05

Splendens

Single

Double

.05

.05

.10

.05
Rochelle Flower Seeds are strictly fresh, new crop-the very best obtainable.

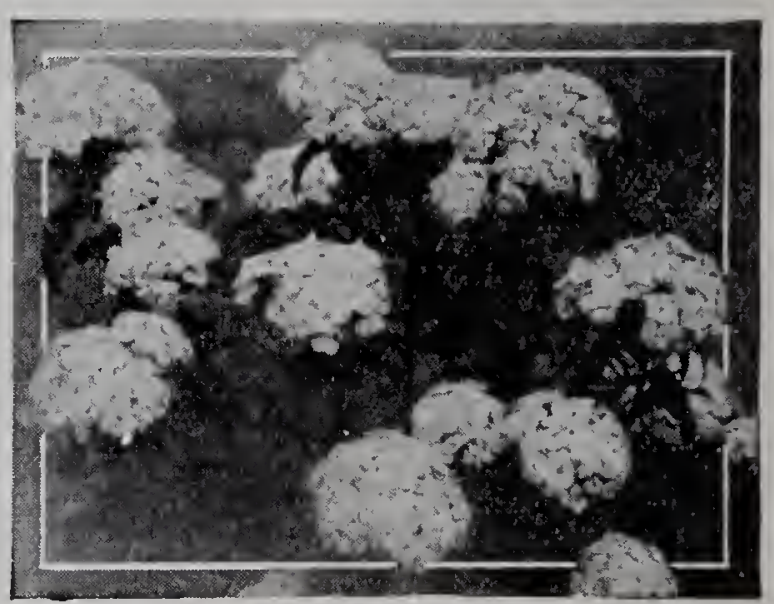

Sweet William

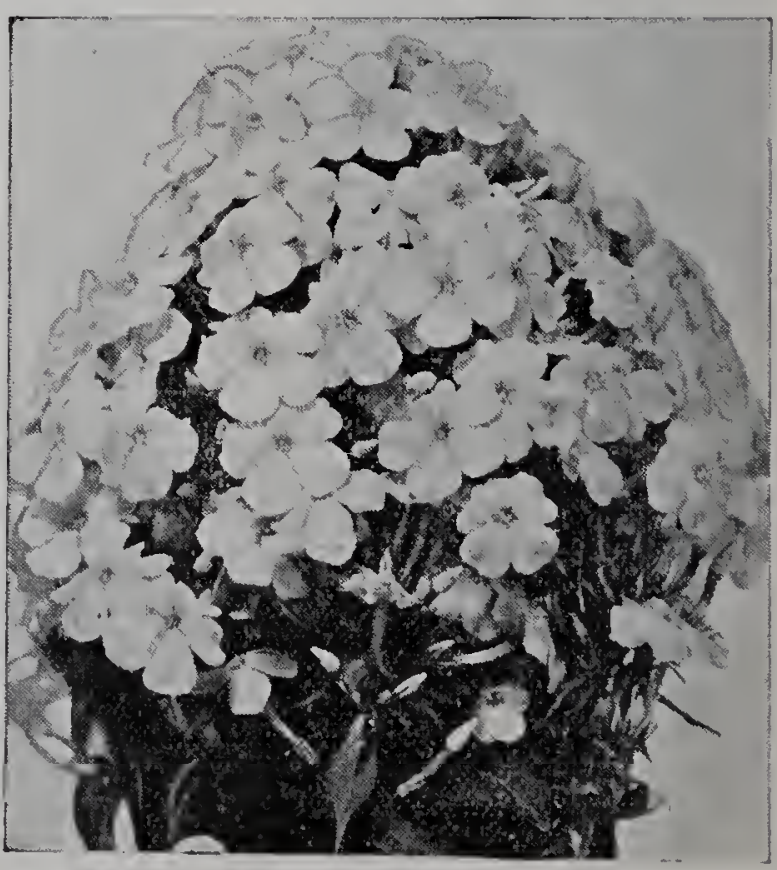

Phlox Drummondi 


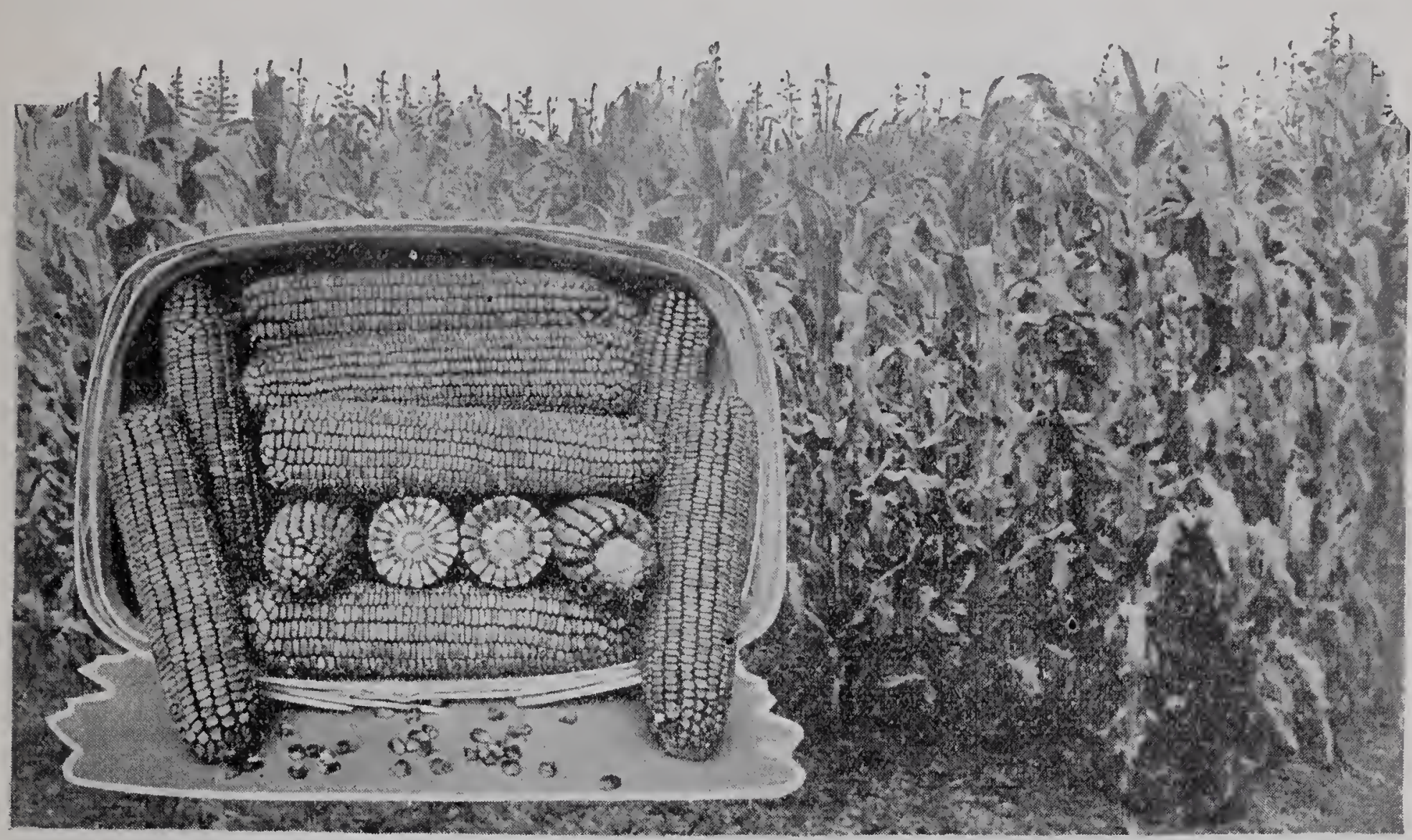

\section{To Our Customers and Friends}

Twenty years ago we began curing Seed Corn for the public. The continued growth of our business and the fact that we are still supplying the same customers that we began with years ago, are ample proofs of the quality of our Seed Corn. We are pleased to announce that we have an extra large supply of good Seed Corn to offer this year. We have also added two new varieties for you to select from.

To new customers we will say that our corn is hand picked from the field and is placed in our curing room before coid weather. All our corn is grown within a radius of five or six miles of Rochelle and we handle no other, therefore we guarantee all corn we put out.

Our prices are as low as is consistent with the quality of corn we have to offer. We wish to impress on your mind that we are quoting you SEED CORN HAND PICKED, hand sorted and cured by our cold air process which we consider far superior to heat drying methods. Each individual ear is inspected, then tipped, shelled and graded ready for the edge drop planter. We do not select fancy ears and sell them at an advanced price. We prefer leaving them in the shelled corn, making that as high grade as possible. Therefore do not ask us for Ear Corn.

Yes, we are still growing and selling seed corn, the best that can be procured for your locality. For 20 years we have been curing seed corn by our own cold air method, and have never failed to produce high germinating seed. The high quality of our Seed Corm and careful attention to our customers interests has made us many friends who come back to us every year, depending on the Rochelle Seed Company to take care of their requirements because they know they are safe.

\section{PRICES}

All Seed Corn Shelled and Graded per bushel \$2.50 F. O. B. Rochelle, Ill., no charge for bags.

\section{OUR GUARANTEE}

Is as strong as any firm can make it. We give ten days in which to test our corn, and if you are not satisfied with it, return it at our expense. We demand the money with the order, but we give every buyer the privilege after he buys anything from us to say whether or not it is worth the money, and if you think not, fire the stuff back to us and get your money. Better still, come to our seed house, you will then see how we handle our corn. We also have many other lines of seed that will interest you.

\section{FREE STORAGE}

Many of our customers order their seed corn in the winter and ask us to hold it until toward spring for them. We are glad to do this and make no charge for same. By this plan you can order your corn in January, have it shipped the latter part of March. You are sure of your seed and do not have to take care of it during the cold winter months when there is danger of it getting damaged by the weather.

We frequently sell out of one or two varieties long before we sell out of the others and by getting your order in early you are sure of not being disappointed. We never substitute varieties. If we are out of what you order we always teil you so and let you decide whether you want your money back or want another variety which we have left.

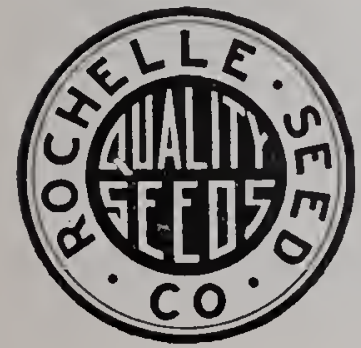

\section{TERMS}

Cash to accompany all Seed Corn Orders. Delivered F.O.B. Rochelle, Illinois. No charge for bags. Ten days given purchaser after delivery of seed to test same, and if test proves below 90 per cent germination, corn may be returned and purchase money will be refunded.

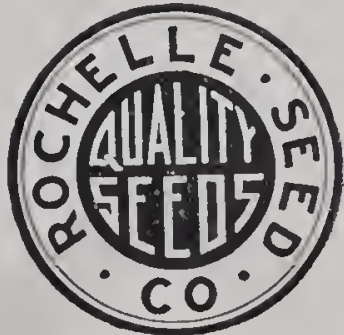




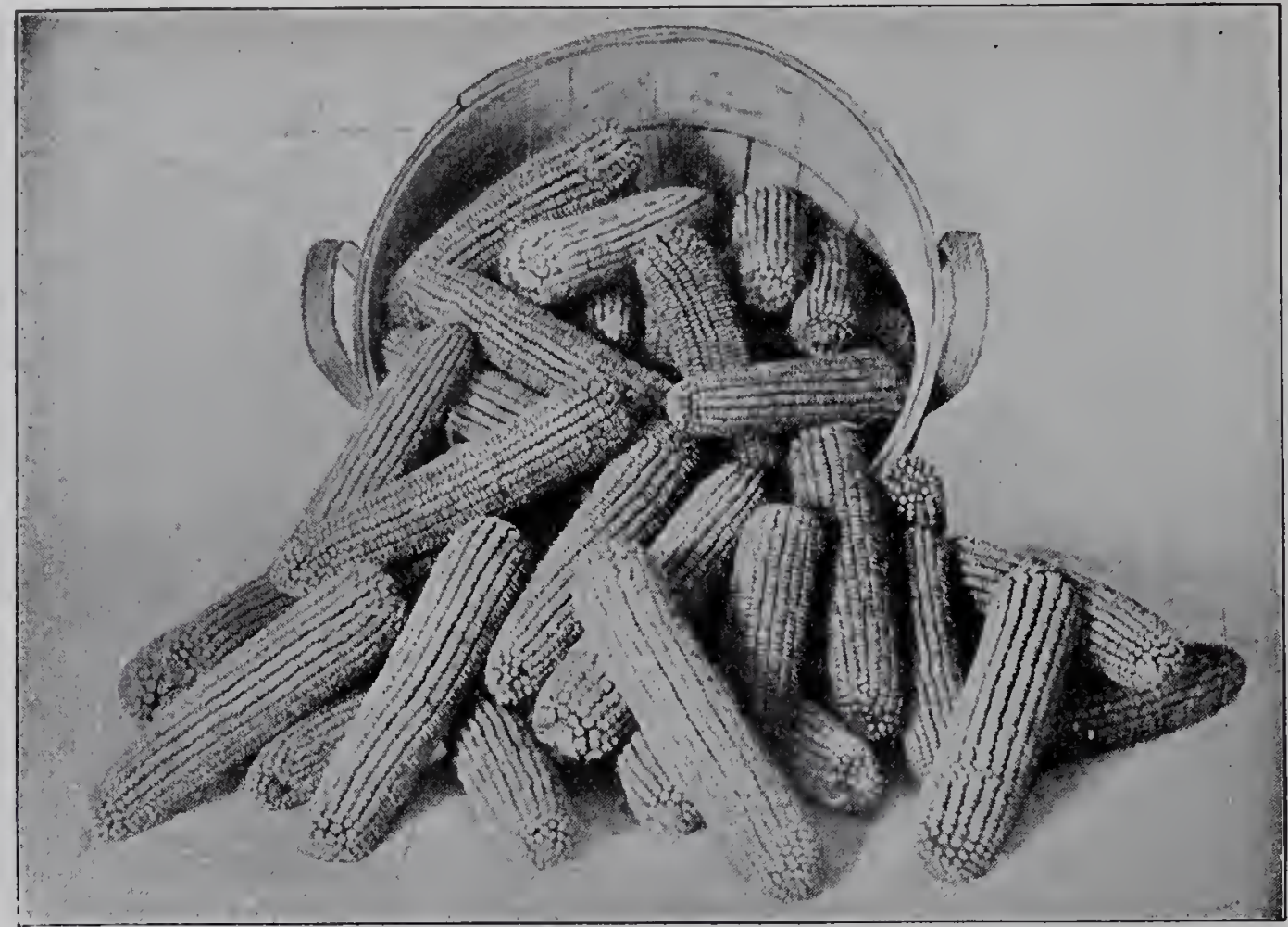

Reed's Yellow Dent Reid's Yellow Dent is unquestionably the greatest variety of corn ever developed for the corn is no longer straight Reid's, but fully ten days earlier than the straight Reid's Corn, having matured this year as early as the Carmichael's Choice and we consider it perfectly safe for this locality. It matures in about 110 days.
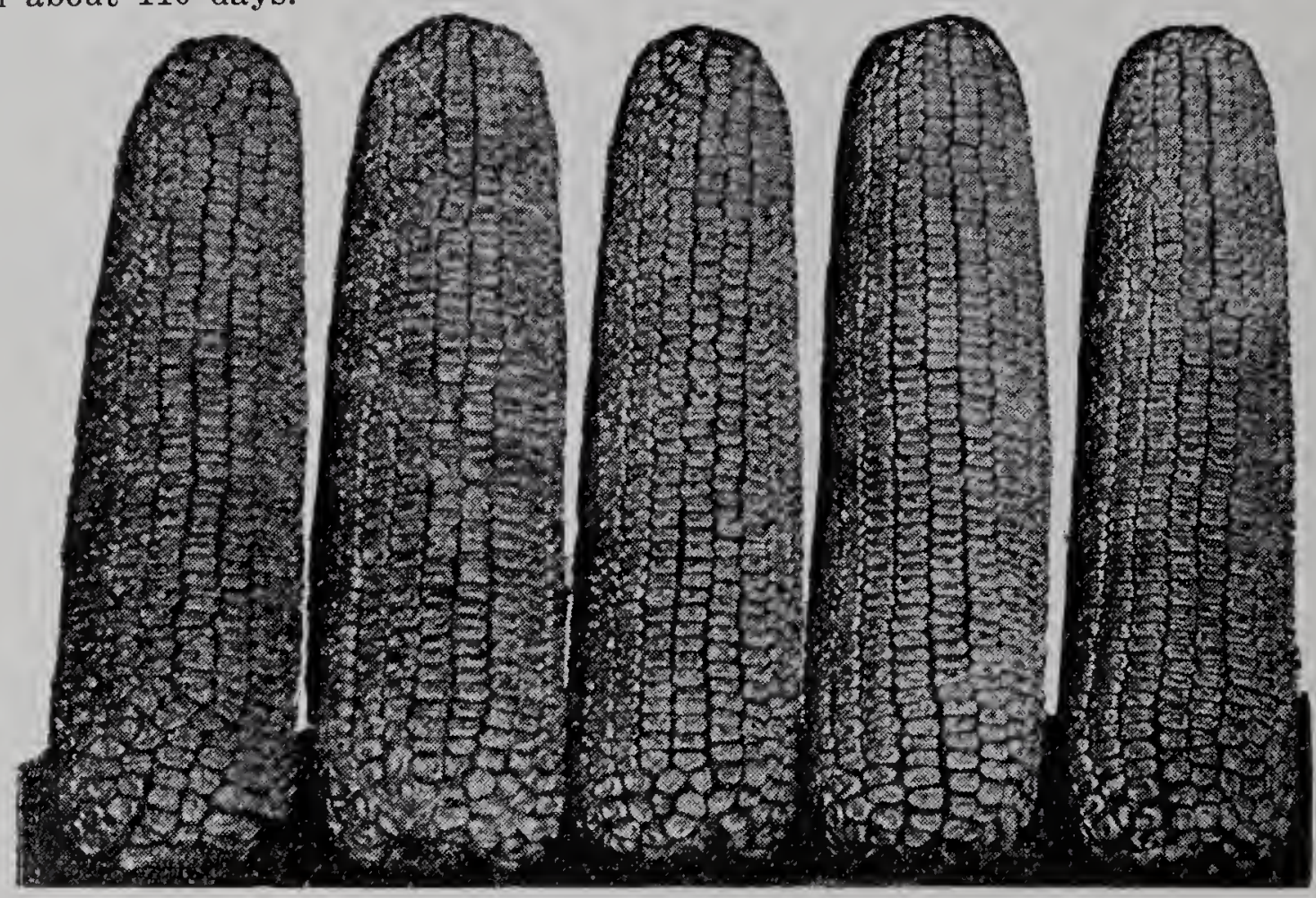

Carmichael's Choice

Carmichael's Choice During the past few years there has been an ever increasing demand for earlier varieties of corn. This we have in Carmichael's Choice. Although it is early, it is by no means small like the early corns. It is very prolific and a hardy grower. The cob is a trifle large but carries a deep grain; will mature easily in 100 days. This is the best shelling corn we know of, and has been grown in this locality for years.

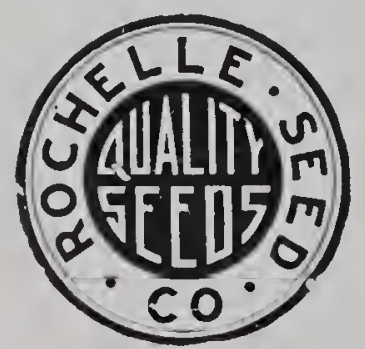

We would be mighty glad if you would give our plant a personal inspection and see for yourself how we handle our seed corn. Many do so each year and state: "I had no idea that you took so much care in getting out your seed corn." Many come expecting to buy ear corn, but after going through and seeing for themselves how it is handled, they will have nothing but shelled and graded seed. They see what they are getting and know it is good. We have nothing to hide. We are proud of our corn. We welcome you to visit our plant and inspect our business. 


\section{Number 10}

If you are looking for a real early corn you will find our Number 10 a few days earlier than Carmichael's Choice; with good corn weather this corn will mature in 90 days. We have many growers who will grow nothing else as they are getting 50 to 60 bushels per acre of sound quality. This is by no means small corn, and a splendid shelling corn, 70 lbs. frequentiy shelling 61 to $63 \mathrm{lbs}$. of the highest grade corn. A general favorite among the feeders, who appreciate a good sound corn.

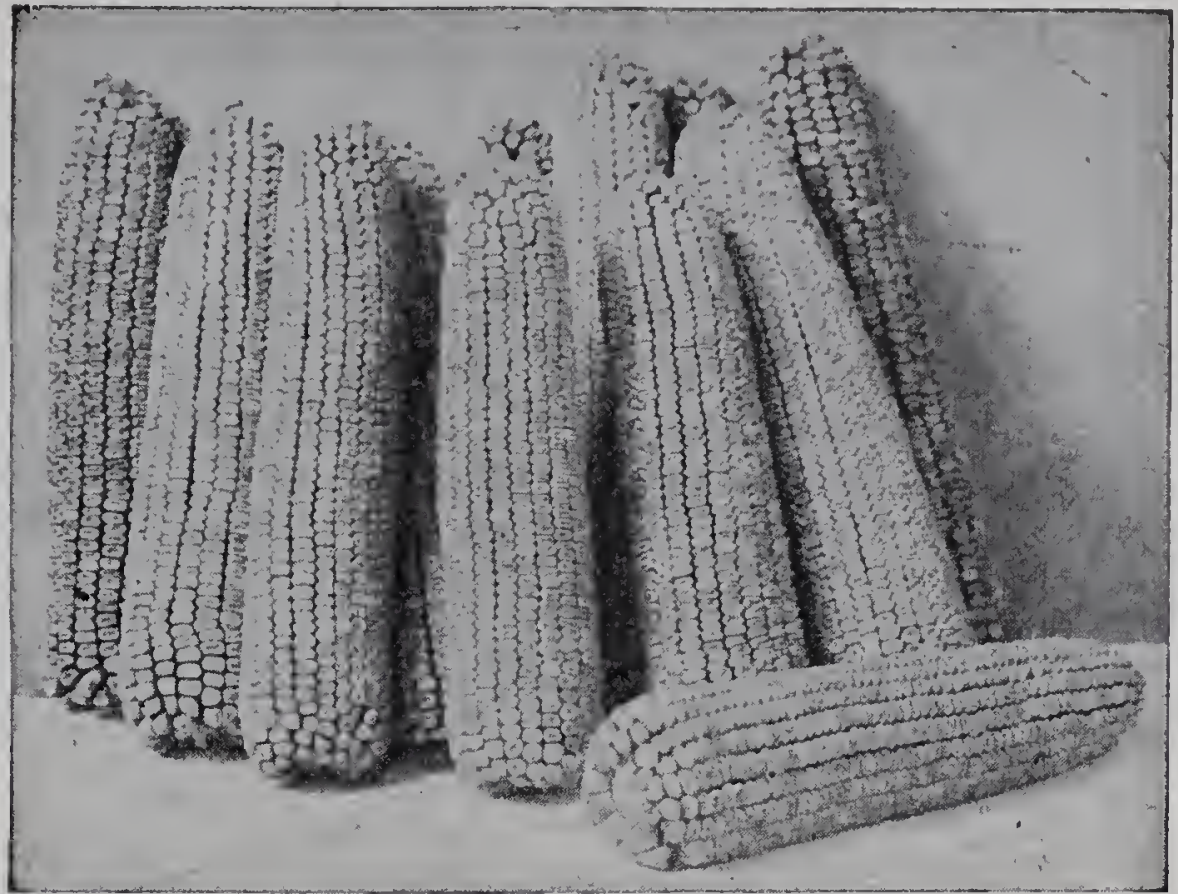

Number $10-$ Yellow

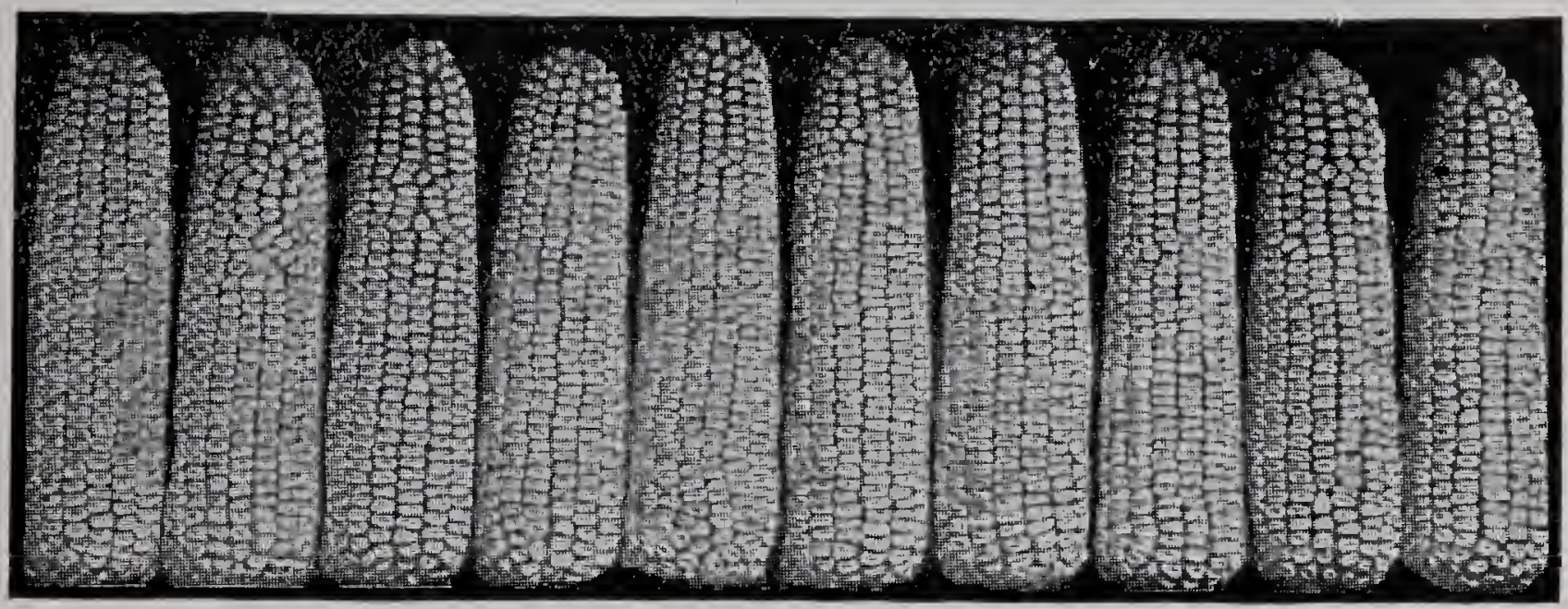

Silver King No. ?

Silver King or No. 7 For a number of years we have been breeding this early white corn in order to meet the demands from those who prefer to grow an early white corn instead of yellow. We have tested this variety of corn both for its early maturity and for its high yielding qualities, to our satisfaction, and do not hesitate to recommand No. 7 to anyone looking for a good early white corn. Original seed came from Agricultural College of Wisconsin several years ago and is thoroughly acclimated and somewhat larger than when we began with it, but it still retains its earliness, maturing in 90 days of good corn weather.

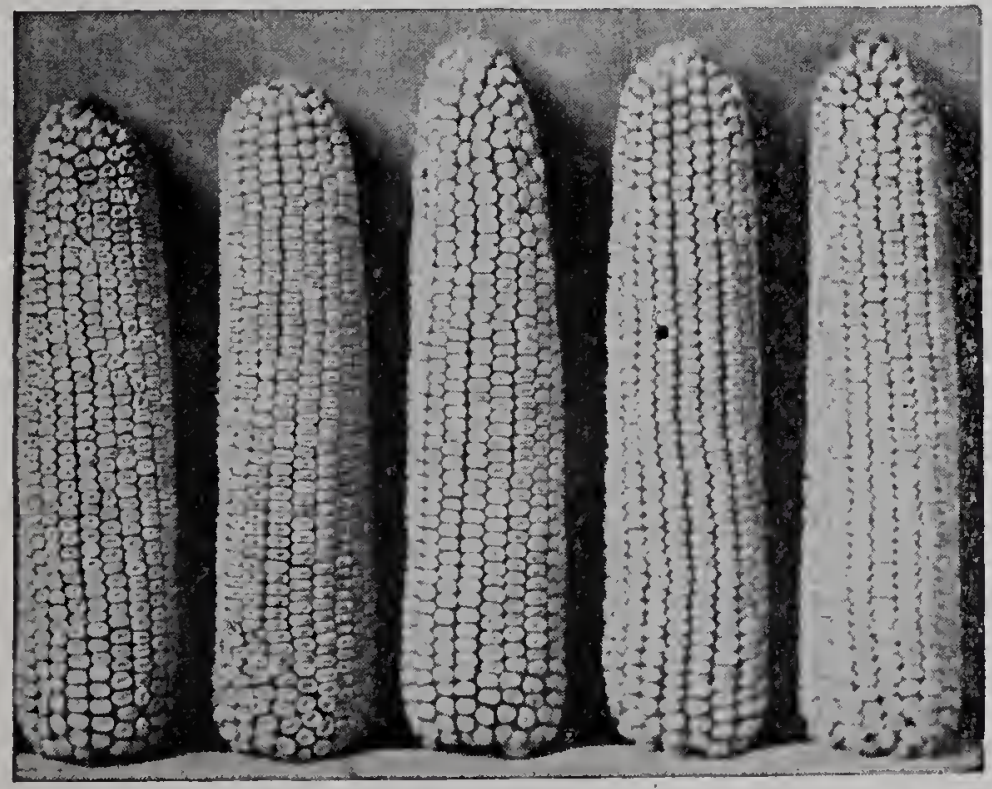

Keck's White

\section{Keck's White}

This variety of corn is too well known among growers of white corn to require any description from us. To those who have never tried our seed will say we know what this corn is. We have tried it under all conditions and always find it "delivers the goods." It will fill the requirements for a profitable white variety as any we have ever raised and we offer it to our customers, knowing that they will find it all we claim. This corn is a vigorous grower, early maturing, cob pure white. Ears are 9 to 11 inches in length, 18 to 20 rows of pure white corn; will get out of way of frost in about 105 days. There is more of the Keck's White grown in this locality than all other white varieties.

Be sure to treat your seed corn with Semesan, Jr. 

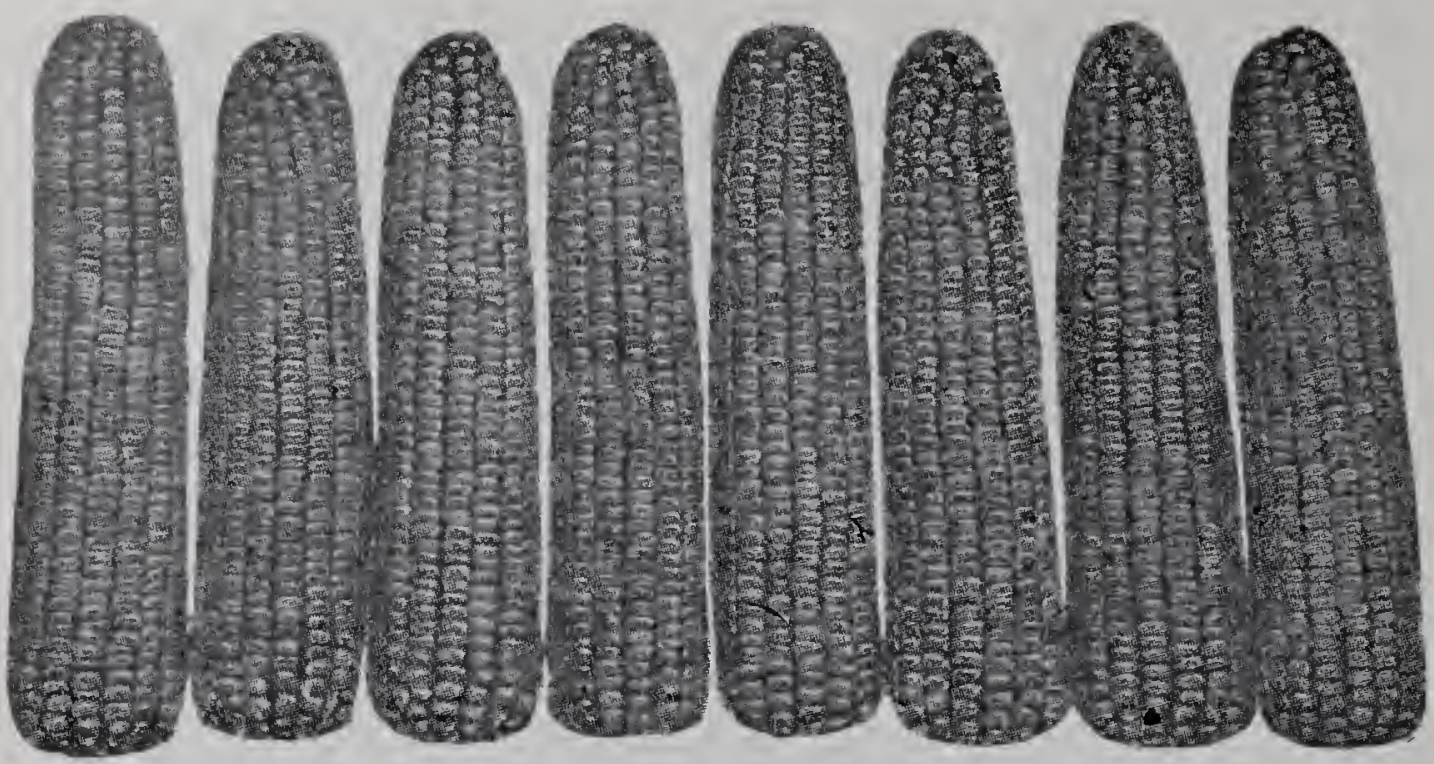

\section{Golden Glow}

This is the famous Golden Glorv of Wisconsin, large variety and cold resisting. Our stock of this famous corn came direct from Wisconsin last year and was grown in Ogle County this year. To those wanting a very early corn for early feeding, we recommend this corn. Matures in about 85 days.

Golden Glow

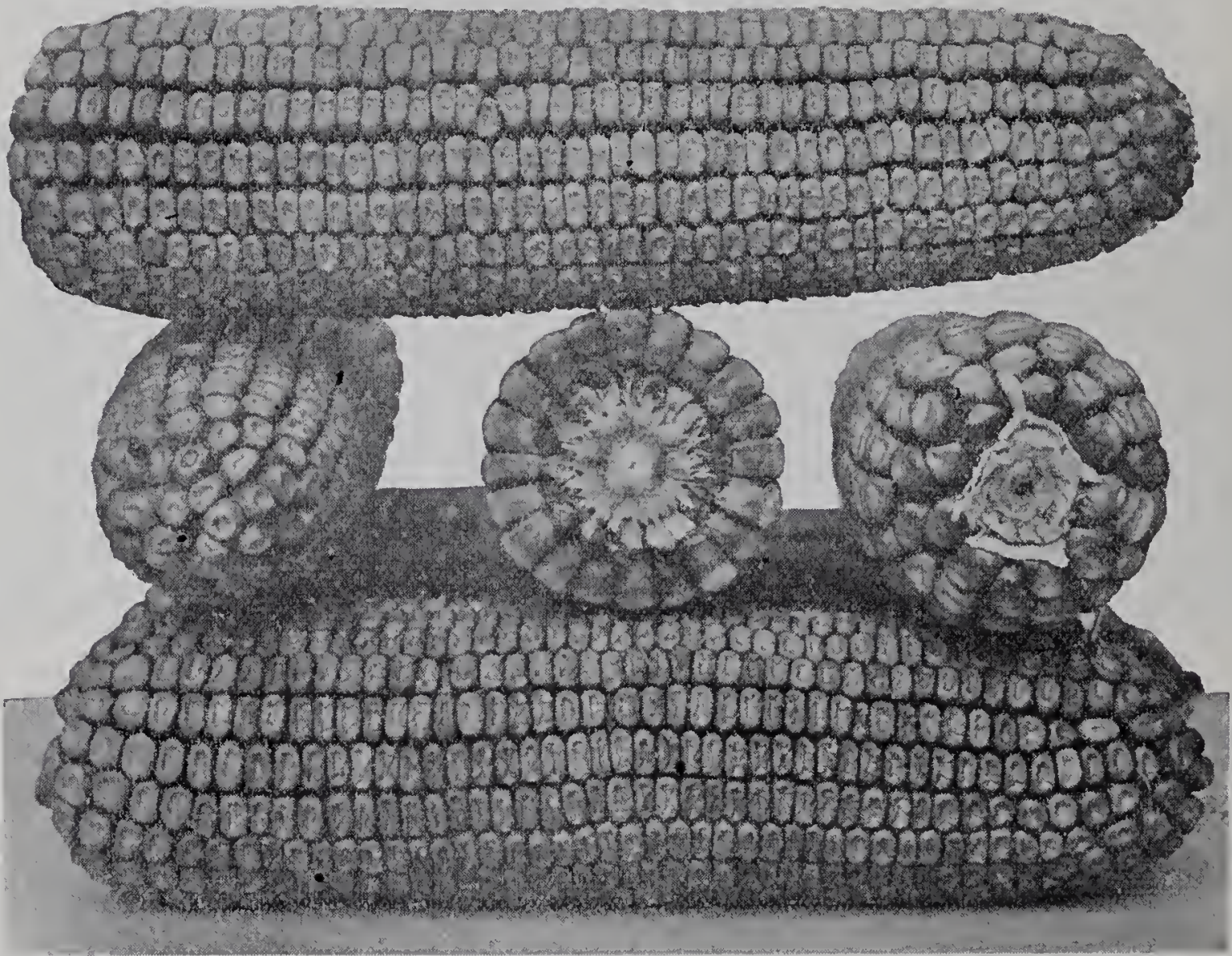

Western Plow man

Western Plowman Western Plowman was originated by Wm. Green and Wm. Webb of Will County. This corn has proven to be one of the best yielding varieties for Northern Illinois. We especially recommend this variety to our Northern Illinois and Iowa customers. It is a deep yellow corn. The ears are more cylindrical than tapering. It has a very high percentage of shelled corn to cob and it matures in about 110 days. We have the pure strain.

\section{Special White Seed Corn Notice!}

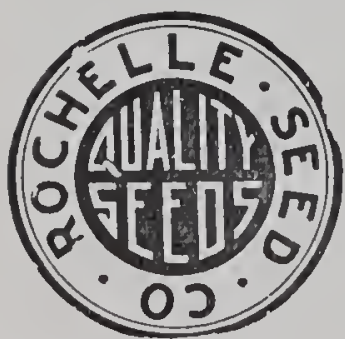

To Our Customers and Friends:

We feel it our duty to help our customers and friends in whatever way possible. White corn has been worth from $2 \mathrm{c}$ to $10 \mathrm{c}$ per bushel on the market over yellow corn of equal grade during the past year. Now it seems to us one should arrange their acreage so as to have at least part white corn, if not all. We have some of the choicest varieties of white seed corn. Give us your order early and you will be sure of having it at planting time. 


\section{Krug's}

Krug's corn was developed by Geo. Krug of Minonk, Illinois. It was selected from a strain of Reid's Yellow Dent. Although this corn has been on the market less than seven years it is the most famous and best advertised corn since Reid's Yellow Dent. It is high yielding, medium maturing, and exceptionally free from disease. We have the pure strain of Krug's yellow dent corn. Grown from certified seed last year. It matures in about 110 days.

Be sure and treat your corn with Semesan Jr.
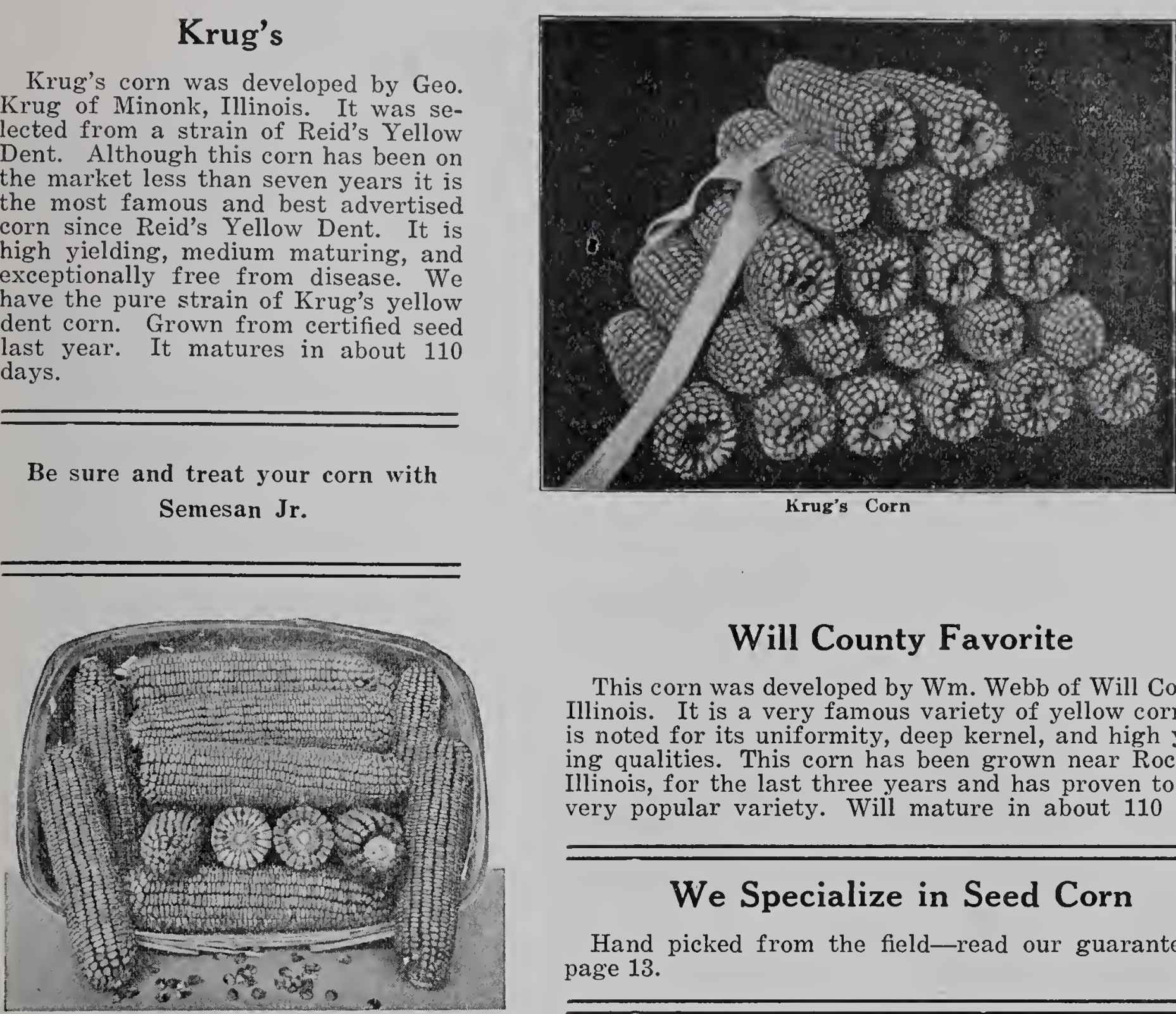

Krug's Corn

\section{Will County Favorite}

This corn was developed by Wm. Webb of Will County, Illinois. It is a very famous variety of yellow corn and is noted for its uniformity, deep kernel, and high yielding qualities. This corn has been grown near Rochelle, Illinois, for the last three years and has proven to be a very popular variety. Will mature in about 110 days.

\section{We Specialize in Seed Corn}

Hand picked from the field-read our guarantee on page 13.

Will County Favorite

\section{SEED CHART}

SEED

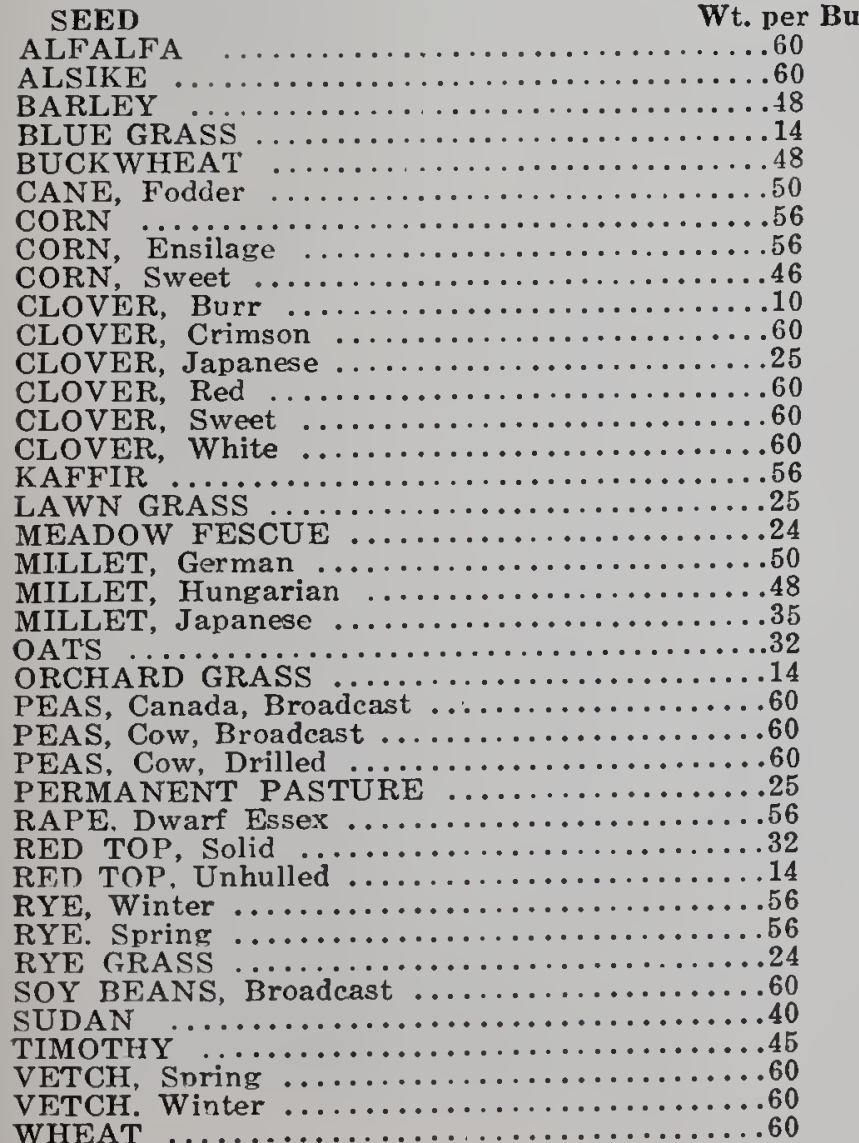

Lbs. per Acre 15-20

$8-10$

$72-96$

$30-35$

$48-60$

60-65

8-10

$30-35$

10-15

$15-20$

$15-20$

$15-25$

$15-25$

20-30

6-8

$20-30$

100-150

25-35

$40-50$

$40-50$

25-40

$50-75$

$20-25$

$90-120$

$90-120$

$60-70$

20-30

6-10

8-12

$20-30$

$70-90$

$50-65$

25-35

$60-90$

$4-25$

$8-12$
$50-70$

$50-70$

$30-50$
$90-120$
Time of Seeding

April, May-August, September February, June-August, November April-June

April, June-August, September May-June

May-June

May-June

May-June

May-July

August, November

July-September

February, May

February, April-August, November April, May-August, September April, June-August, October May-June

April, June-August, October April, May-August, September May-July

May-July

May-July

March-May

April, May-August, September February, April

May-August

May-August

April, June-August, October

April-August

Apri- June-September, November A pril, June-September, November September-November

March-May

April, June-August, October May-August

April-June

April. June-August, November

March-May

August-November

September-October 


\section{FARM SEEDS}

Important! The prices named herein on Farm Seeds are those prevailing at the time this Catalog goes to press and are subject to market fluctuations. As values usually advance on many items during the season, it will be to your advantage to order early. While we reserve the right to change these prices when the market warrants a change, we shall maintain these prices as long as possible.

BARLEY

Oderbrucker Type Barley. Write for prices.

Velvet Barley. Write for prices.

CANE SEED or SORGHUM-10 lbs. or less, $10 \mathrm{c}$ per $\mathrm{lb}$. postpaid. Containers at value. Write for prices on larger quantities.

KAFFIR CORN-10c per lb. postpaid. Write for prices on larger quantities. Containers at value.

SEED WHEAT-SPRING

New Progress. Write for prices.

DWARF ESSEX RAPE-1 lb. 15c, 5 lbs. 65c, $10 \mathrm{lbs}$. $\$ 1.25$ postpaid. Buyers expense $100 \mathrm{lbs} . \$ 9.00,25 \mathrm{lbs}$. or over filled at 100-lb. rate. For $25 \mathrm{lbs}$. or under add 15c for cotton bags, larger quantities add $35 \mathrm{c}$ for grain bags.

SOY BEANS. Write for prices.

Manchu.

Illini.

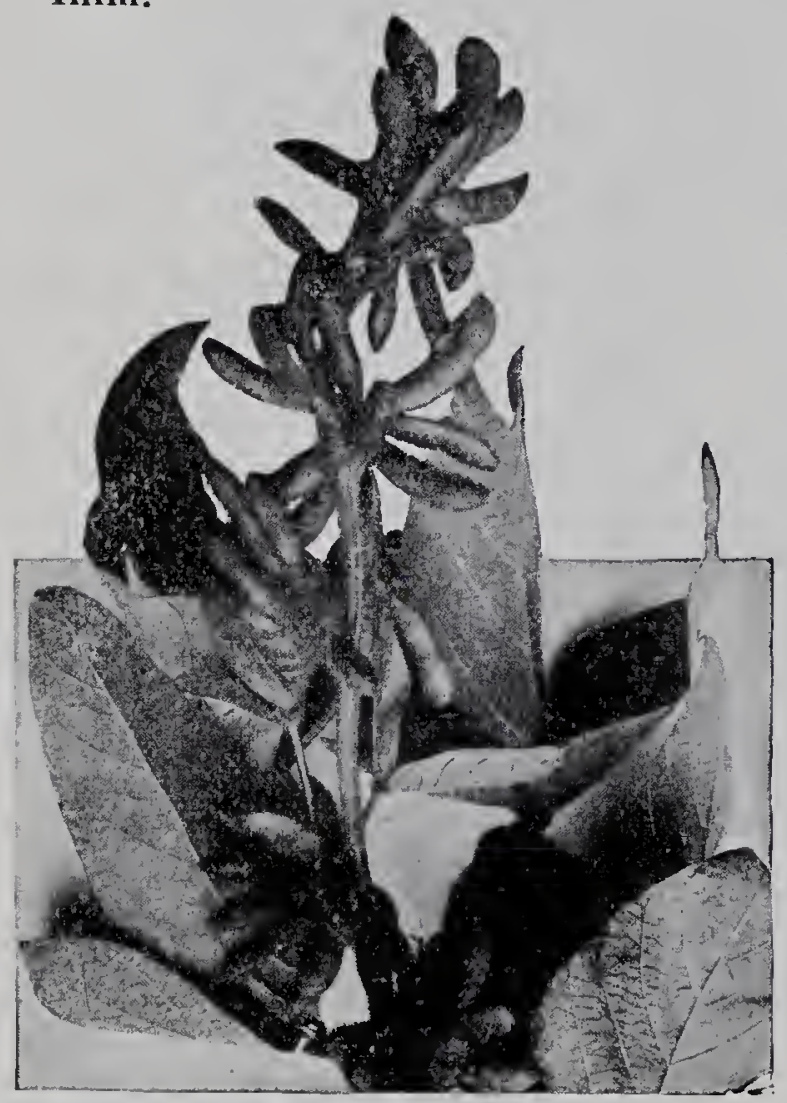

ALFALFA

North w e tern Grown.

Write for prices.

Montana Regis-

tered $\mathrm{G}$ r i m m.

Write for prices.

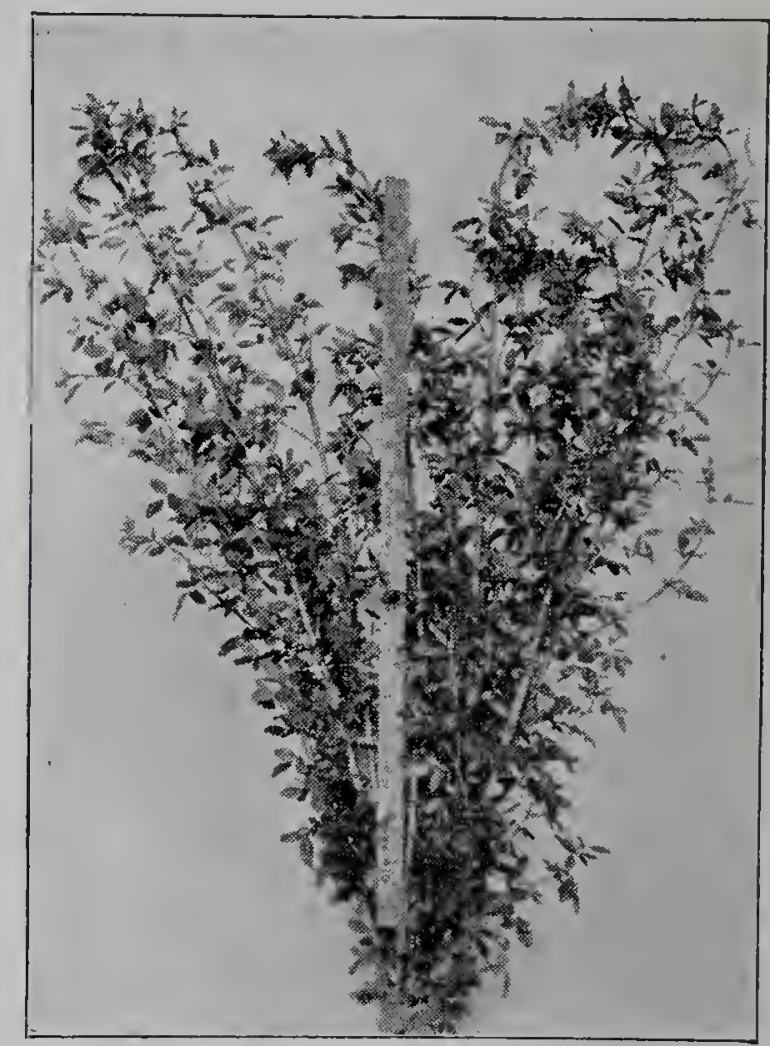

Northwestern Grown Alfalfa

CLOVER SEEDS-Write for prices.

Grundy County Sweet Clover.

Tall White Blossom Sweet Clover.

Yellow Blossom Biennial Sweet Clover.

Alsike Clover.

Medium Red Clover.

Mammoth Red Clover.

Be sure and use Nodogen.

\section{Cyclone Seed Sower $\$ 2.00$ Each}

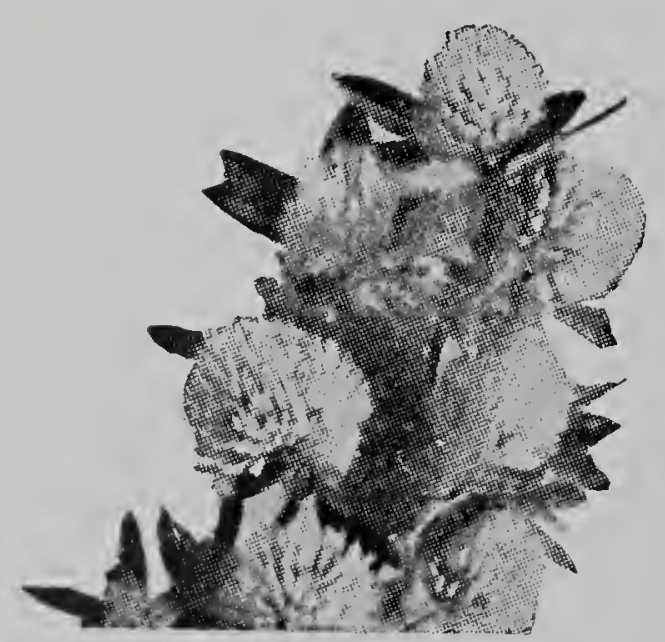

Medium Red Clover
GRASS SEEDS-

Write for prices.

Timothy

Millet

Sudan Grass

WINTER RYE-

Write for prices.

WINTER WHEAT

-Write for prices.

Kanred

OATS-Write for prices.

Iowar White Russian Yellow

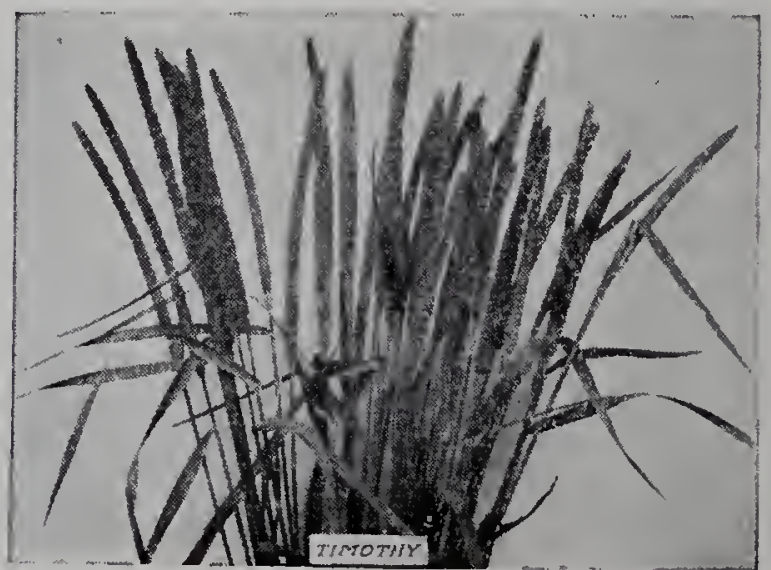




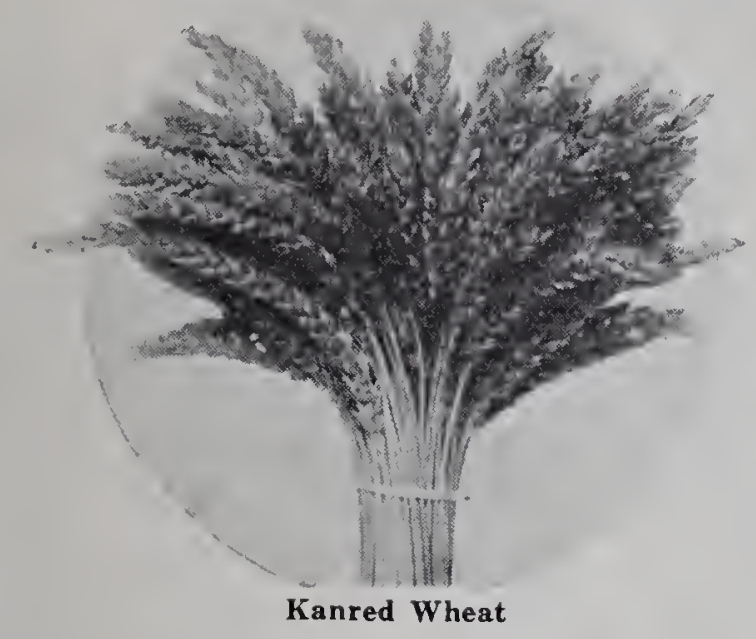

\section{Nitragin Legume Inoculator}

All Clovers- $1 / 2$ bu. size. $\$ .60$

1 bu. size.

5 bu. size. 4.75

Soy Beans-

1 bu. size.

2 bu. size

5 bu. size 1.50

10 bu. size 2.50

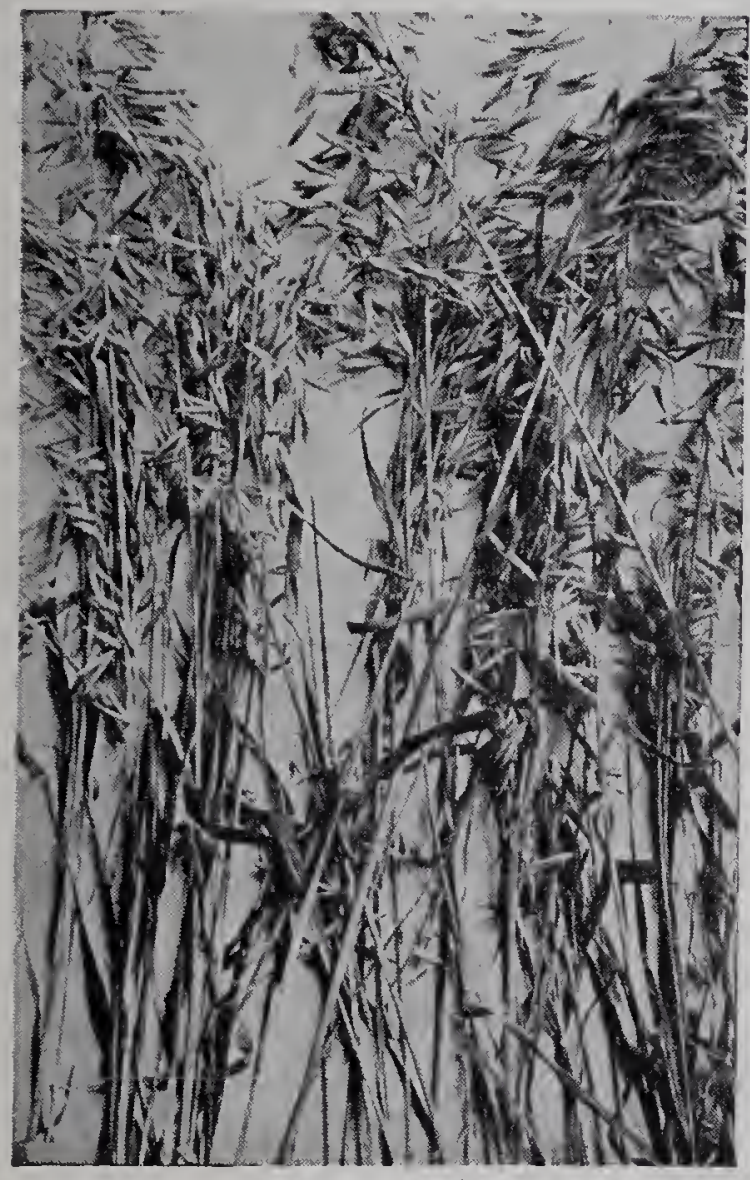

Iowar White Oats
Nodogen

JELLY AND HUMUS DRY FORM

Inoculation for all Clovers, per bu... $\$ .50$

Inoculation for Soy Beans, per bu

Inoculation for Soy Beans, five bushels

35 1.25

\section{Lawn Grass}

IDEAL LAWN GRASS-1 lb. 35c, 5 lbs. \$1.50, postpaid. Ask for prices on larger quantities.

WHITE DUTCH CLOVER-1/2 lb. 30c, $1 \mathrm{lb}$. 55c postpaid. Ask for prices on larger quantities.

KENTUCKY BLUE GRASS-35c per lb. postpaid. Ask for prices on larger quantities.

RED TOP GRASS-35c per lb. postpaid.

\section{Notice!}

\section{OUR MOTTO-PROMPT SERVICE}

Remember when you place your order with the ROCHELLE SEED CO. for garden and flower seeds, your order will be filled and mailed to you the same day we receive it.

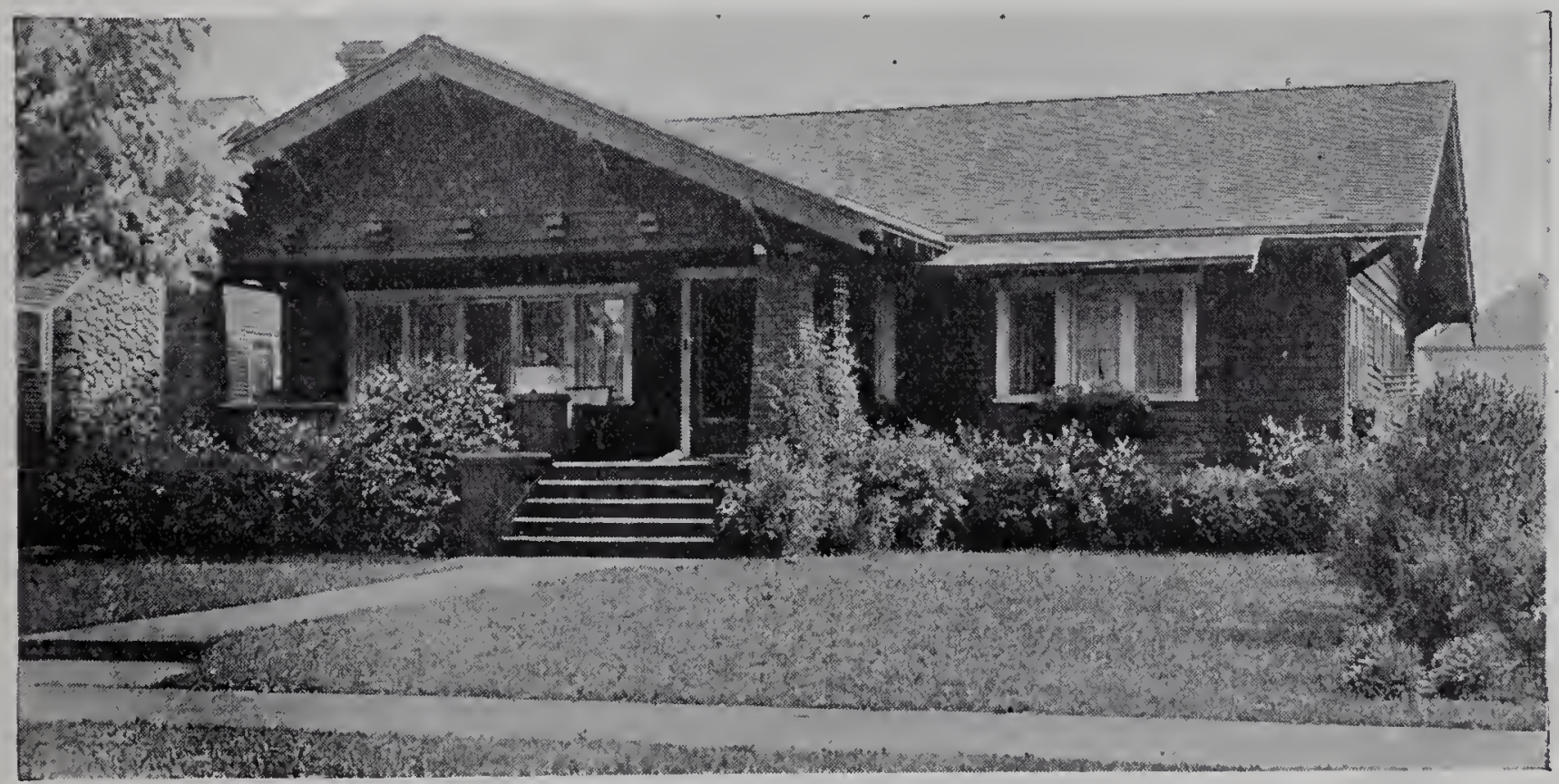

Have a Beautiful Lawn-Sow Rochelle Quality Seed 


\section{DU BAY SEED DISINFEGTANTS}

CERESAN -A dust disinfectant for Wheat, Oats, Barley and certain other cereals. 8-0z. tin 50c, 1 -lb. tin $75 \mathrm{c}, 5-\mathrm{lb}$. tin $\$ 3.00,25-\mathrm{lb}$, pail $\$ 12.50$, 100 -lb. drum $\$ 49.00,300-1$ b. drum $\$ 144.00$.

SEMESAN-A general disinfectant for many vegetable and flower seeds or bulbs, and certain plant diseases. 2-oz. tin 50c, $1-1 b$. tin $\$ 2.75,5-1 b$. tin $\$ 13.00,25-1 b$. pail $\$ 56.25,100-1 b$. drum $\$ 220.00,300-1 b$. drum $\$ 645.00$.
SEMESAN JR.-A dust disinfectant for Seed Corn. 4-oz. tin 50c, $1-1 b$. tin $\$ 1.75,5-1 b$. tin $\$ 8.00$, 25-lb. pail $\$ 31.25,100-1 b$. drum $\$ 120.00,300-$ lb. drum $\$ 345.00$.

SEMESAN BEL-An instantaneous dip disinfectant for Seed Potatoes. 4-oz. tin 50c, 1-lb. tin $\$ 1.75$, 5-lb. tin $\$ 8.00 ; 25-1 b$. pail $\$ 31.25,100-1 b$. drum $\$ 120.00,300-1 b$. drum $\$ 345.00$.

DU BAY SEED DISINFECTANTS are poisonous and owing to present Postal Regulations cannot be sent through the mails but must be sent by express or freight.

PHENOL, the best known animal germicide. As a healing powder it has no equal. 1-lb. can 25 c, $25-\mathrm{lb}$. pail $\$ 3.50,100$ lbs. $\$ 7.00$.

WIZARD BRAND SHEEP MANURE-Prices postpaid. 2-lb. carton $20 \mathrm{c}, 5$-lb. carton $35 \mathrm{c}, 10-\mathrm{lb}$. carton $65 \mathrm{c}, 25-\mathrm{lb}$. bag $\$ 1.00,50-\mathrm{lb}$. bag $\$ 1.90$. Packages called for at Seed House or sent by freight at the following prices: 25 -lb. bags $70 \mathrm{c}, 50$-lb. bags $\$ 1.35$.

VIGORO-Specially prepared plant food. 25 lbs. $\$ 1.75,50$ lbs. $\$ 3.00$, buyer's expense.

WE HANDLE ARMOUR'S BIG CROP COMMERCIAL FERTILIZER. Ask for prices.

SODIUM CHLORATE_For extermination of Canada Thistle, Quack Grass, Wild Morning Glory, Poison Ivy and Burdock. Ask for prices.

\section{BARBAK}

Prices for Barbak 111 Super Corn Seed Disinfectant

$4 \mathrm{oz}$. ..$\$ 50$

$1 \mathrm{lb}$. 1.75

5 lbs. .................. 8.00

25 lbs. ................... 31.25

100 lbs. ................... 120.00

300 lbs. ................... 345.00

\section{$25 \mathrm{c}$ COUPON \\ Profit Sharing Coupon \\ Worth 25 Cents on All Orders of \$2.00 or Over \\ IF YOUR ORDER FOR \\ Vegetable and Flower Seeds \\ $25 \mathrm{c}$ \\ COUPON}

Amounts to $\$ 2.00$ or Over Deduct 25c for this Coupon

For this Coupon and $\$ 1.75$ you may select Vegetable or

Flower Seeds to value of $\$ 2.00$

Be sure to enclose this profit-sharing coupon with your order if you deduct the 25 cents

This Coupon is Also Good if Presented at Our Store

Rochelle Seed Company

ROCHELLE, ILLINOIS 


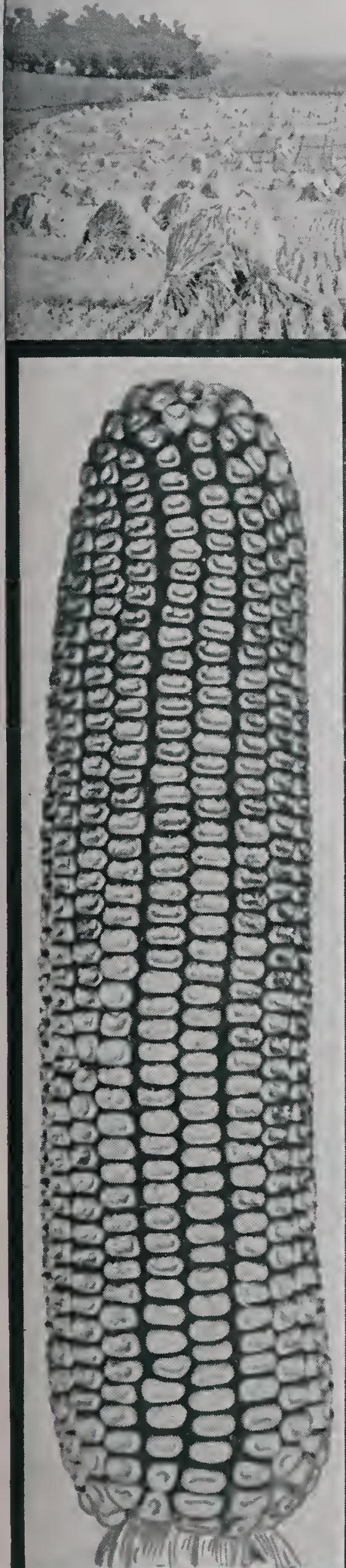

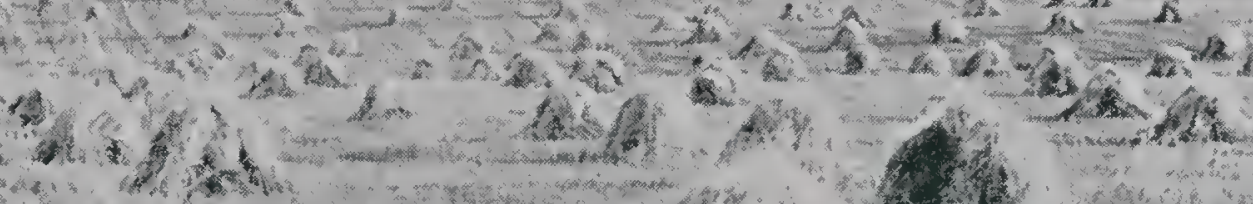

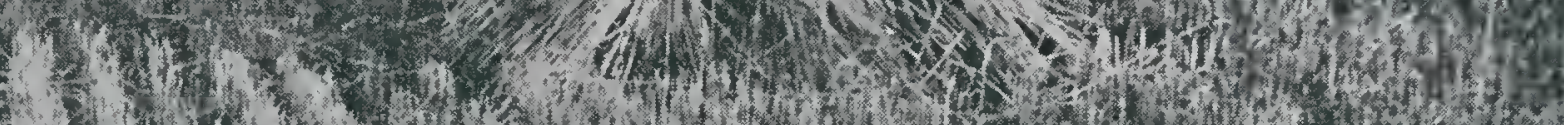
1
$y$

\section{Get Our Prices on Field Seeds and Grasses}

We are wholesaler's and retailer's of clover seed of all kinds, including timothy seed, soy beans, wheat, corn, oats, barley and rye. You will do well to obtain our prices before buying elsewhere and if you have any of the above will do equally as well to get our prices on same, as we pay top prices on seeds and grain at all times, quality considered.

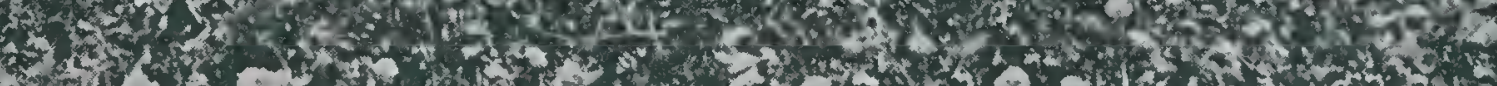

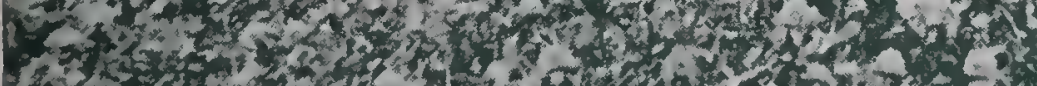

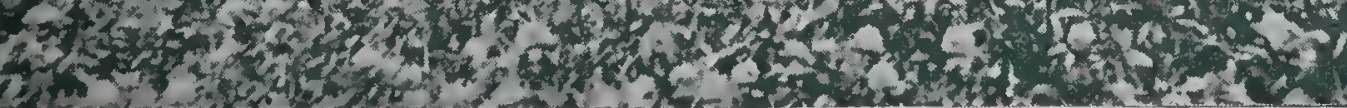




\section{FIELD AND GARDEN}

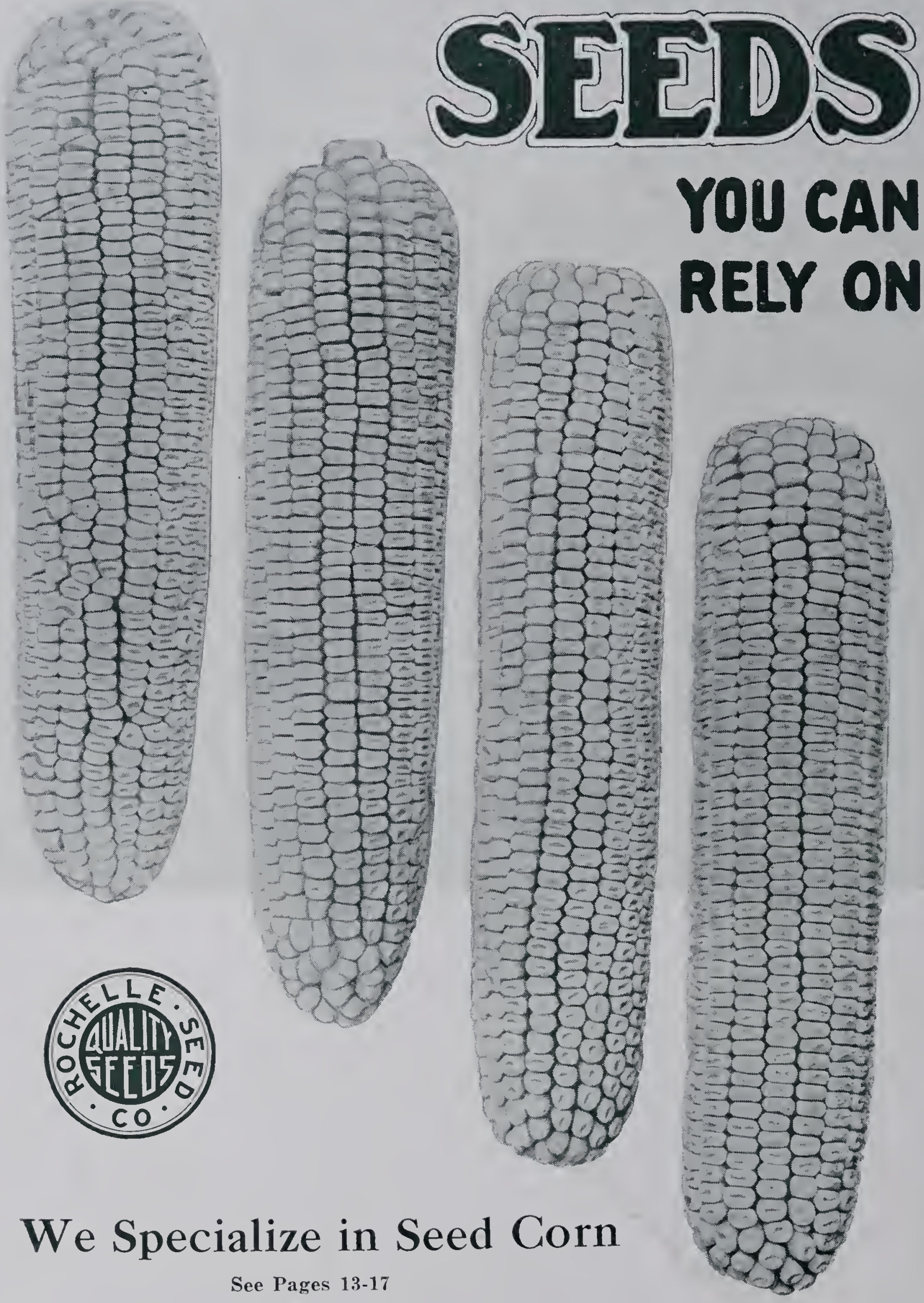

